IZA DP No. 10035

Trade Induced Skill Upgrading:

Lessons from the Danish and Portuguese Experiences

Grace (Weishi) Gu

Samreen Malik

Dario Pozzoli

Vera Rocha

July 2016 


\title{
Trade Induced Skill Upgrading: Lessons from the Danish and Portuguese Experiences
}

\author{
Grace (Weishi) Gu \\ University of California Santa Cruz \\ Samreen Malik \\ New York University Abu Dhabi \\ Dario Pozzoli \\ Copenhagen Business School and IZA \\ Vera Rocha \\ Copenhagen Business School and IZA
}
Discussion Paper No. 10035
July 2016

IZA

P.O. Box 7240

53072 Bonn

Germany

\author{
Phone: +49-228-3894-0 \\ Fax: +49-228-3894-180 \\ E-mail: iza@iza.org
}

\begin{abstract}
Any opinions expressed here are those of the author(s) and not those of IZA. Research published in this series may include views on policy, but the institute itself takes no institutional policy positions. The IZA research network is committed to the IZA Guiding Principles of Research Integrity.

The Institute for the Study of Labor (IZA) in Bonn is a local and virtual international research center and a place of communication between science, politics and business. IZA is an independent nonprofit organization supported by Deutsche Post Foundation. The center is associated with the University of Bonn and offers a stimulating research environment through its international network, workshops and conferences, data service, project support, research visits and doctoral program. IZA engages in (i) original and internationally competitive research in all fields of labor economics, (ii) development of policy concepts, and (iii) dissemination of research results and concepts to the interested public.
\end{abstract}

IZA Discussion Papers often represent preliminary work and are circulated to encourage discussion. Citation of such a paper should account for its provisional character. A revised version may be available directly from the author. 
IZA Discussion Paper No. 10035

July 2016

\section{ABSTRACT \\ Trade Induced Skill Upgrading: \\ Lessons from the Danish and Portuguese Experiences}

We study how the skill distribution for an economy responds to changes in the skill premium induced by trade integration. Using administrative data for Denmark (1993-2012) and Portugal (1993-2011), we conduct a two-step analysis. In the first step we predict the skill premium changes which are triggered by exogenous trade shocks. In the second step we estimate the impact of such changes on the skill distribution. The main results for Denmark show that both the average and the standard deviation of skills increase as a result of trade integration. For Portugal we find instead that the impact of trade mediated by skill premium changes is negligible and not statistically significant. We provide a theoretical intuition to rationalize both sets of results.

JEL Classification: F16, J24

Keywords: $\quad$ skill premium, skill upgrading, trade integration, labor market frictions

Corresponding author:

Dario Pozzoli

Department of Economics

Copenhagen Business School

Porcelaenshaven $16 \mathrm{~A}$

DK-2000 Frederiksberg

Denmark

E-mail:dp.eco@cbs.dk 


\section{Introduction}

Traditional trade models with endogenous human capital acquisition provide strong predictions about skill upgrading patterns: opening up to trade induces skill upgrading for countries with a large endowment of skilled workers whereas unskilled abundant countries experience a skill downgrade. These strong predictions are at odds with recent theoretical and empirical research that has highlighted that with in an economy globalization may have heterogeneous effects on workers skill upgrading decisions: some workers may respond to globalization by increasing human capital investments while others may cut back on skill upgrading (see e.g., Edmonds et al., 2009; Hickman and Olney, 2011; Atkin, 2012; Blanchard and Willmann, 2016).

Workers' heterogeneous skill upgrading in response to trade integration suggest that globalization may have important implications for the skill distribution of a country. Specifically, trade may potentially change not only the average (mean) but also the diversity (variance) of skills. A potential transmission channel could be that trade has a positive effect on wages associated with certain skills but a negative effect on wages of other skills resulting in a wage gap across skills (henceforth "skill premium"). A change in skill premium may in turn affect individuals' incentives to invest in skill upgrading heterogeneously. In particular, individuals who face an increase in the skill premium, will most likely upgrade their skills if the return from new skills exceed the cost of skill acquisition. The opposite holds true for individuals who experience a reduction in skill premium. Consequently, a non-monotonic response of skill upgrading decision at an individual level can impact the entire domestic distribution of skills.

The degree with which the above transmission channel affects the entire skill distribution depends on whether individuals can freely adjust their skills in response to changes in skill premium. Domestic policies and institutions therefore can be integral in this adjustment process. For example, if domestic regulations are conducive to smooth functioning of labor markets, such as a flexible employment protection legislation and a liberal educational policy, then one can expect that trade shocks to skill premium are more likely to affect the skill composition within the economy. However, such effects may in fact be absent under a frictional setting.

In this paper we formally study the effect of changes in skill premium induced by trade integration (henceforth "trade-induced skill premium") on domestic skill distribution and how such effects interact with domestic labor market frictions. A systematic study highlighting these effects can enhance our understanding of economic and policy implications of trade 
integration. However, such distributional effects are not extensively studied in the existing literature. On the one hand, Bombardini et al. (2012) highlight that distribution of skills (that is not only the average endowment of skills but the variation of skills) within an economy is a crucial predictor of export volumes in a standard gravity equation model. On the other hand, our analysis highlights the issue of reverse causality of trade integration where trade in itself may impact the distribution (the mean and the variance) of skills. Therefore, our work shows that the distributional effects in response to trade should be accounted for while designing trade and other domestic policies.

To provide an intuition of how individual level decisions for skill upgrading (in response to changes in skill premium) impact the entire domestic skill distribution, we present a simple 3 period model. In the model, each individual is characterized by her innate ability and is endowed with a fixed amount of time. In each period she decides whether to allocate her endowed time towards work or skill upgrading. If she works, she earns a wage otherwise, she bears an opportunity cost of missed wages but better skills enhance her wages for the remaining periods. The individual ultimately maximizes her lifetime utility function subject to her budget constraint. The optimal decision rule uses thresholds of exogenous innate ability, which consequently determine whether the individual upgrades skills once, twice, or not at all in her life time. An exogenous change in the wage gap across various skills (skill premium) affects these thresholds and subsequently affect individual's decision to upgrade skills. In aggregation these individual level decisions impact the overall distribution of skills in an economy. We extend this set-up to encompass the role of labor market frictions to study how frictions can affect the response of the skill distribution to exogenous changes in the skill premium.

Our corresponding empirical analysis is conducted in two steps. In the first step, we estimate the effect of trade integration on the skill premium, proxied by measures of wage gap. In the second step, we assess how changes in the skill premium as predicted from the first step, affect the average and the variance of skills within the economy. We apply this two-step approach to administrative data for Denmark and Portugal which are chosen for the following reasons.

Both economies are industrialized countries characterized by a small, export oriented economy. In terms of trade activity, Denmark and Portugal export almost half of their GDP to other markets (OECD, 2013, 2014). Traditionally Danish and Portuguese trade has been concentrated with a few trading partners but more recently both countries have also started to trade with emerging economies, such as China and formerly communist countries. Thus despite the maturity of these two economies, the process of trade integration is still 
evolving. Also in terms of export composition, the two economies are fairly comparable as both countries have a large export shares within "traditional" sectors, such as machinery equipment, textiles, food and transport (OECD, 2013, 2014).

However these economies are extremely different in terms of labor market institutions and educational policies. On the one hand, Denmark provides abundant subsidies for individuals to upgrade skills. The support is not only in terms of formal schooling but also through comprehensive active labor market policies that aim at keeping the skills of the workforce updated (Andersen, 2012). Moreover Denmark has an extremely flexible labor market which reduces the frictions hindering labor reallocation across firms within industries or across industries. On the other hand, Portugal is characterized by one of the most rigid labor markets in the world (Botero et al., 2004), with less generous educational policies and a milder emphasis on lifelong learning opportunities.

In our empirical analysis, we therefore expect the effects of changes in trade-induced skill premium to be stronger for the Danish skill distribution relative to the Portuguese skill distribution. The comparison across these economies facilitate assessing whether the impact of trade integration on skill distribution is further affected due to varying frictions in the labor market and educational policies across economies. In addition, both countries have documented detailed data entries and provides us with an incredibly rich and comparable empirical environment to study our question of interest.

Our research question described above poses two main challenges from an empirical point of view. First individuals' skills, our variable of interest, are generally very difficult to measure. Second, reverse causality between our measures of skills and trade poses threat to identification. In this paper, we tackle these two important issues as follows.

First, since we base our main measure of skills on workers' wages, such measures may not only reflect the observable and the unobservable features of individuals' skills but also firm level wage policies. To deal with this issue, we disentangle wages into the following four components by using Abowd et al.'s 1999 model: i) a systematic component related to observable workers' characteristics, ii) an unobservable time-invariant component at the worker level, iii) firm fixed effects and iv) a residual. For the purpose of our study and similarly to Iranzo et al. (2008) and Irarrazabal et al. (2013), we focus on the observable features of wages as a comprehensive measure of both formal and informal skills. In general such detailed information on the individual determinants of skills - such as wages and the workers' observables in general - are often difficult to collect for the whole workforce due to confidentiality and privacy laws. ${ }^{1}$ This has limited the analysis in most of the existing

\footnotetext{
${ }^{1}$ See (Goldberg and Pavcnik, 2007) for data limitations.
} 
literature to years of education as a primary measure of skills (see e.g., Blanchard and Olney, 2015; Atkin, 2012). The use of confidential employer-employee linked Danish and Portuguese data allows us to use a more comprehensive measure of skills as the component of wages which is explained by workers' observable characteristics and not only by education levels. To test the robustness of this definition, we also use other measures of skills, as explained later.

To address the reverse causality issue, we apply the same identification strategy as in Hummels et al. (2014) in our first step of empirical model. We tease out the impact of the export (or import) volumes on the skill premium at the industry level, by exploiting the shocks to the world import (or export) demand (or supply). This step provides us with estimates which we employ to predict the trade-induced skill premium. Our second step may also suffer from simultaneity issues given that it is difficult to rule out that the first two moments of the skill distribution do not affect the trade-induced skill premium. To limit this issue we lag the predicted trade-induced skill premium from the first step, as skill premium changes from previous periods are less likely to be driven by the current skill endowments and the variation of skills.

Our main results for Denmark show that trade integration has a non-linear effect on wage gap across various skills and that these changes in the skill premium significantly influence the skill distribution. Specifically, we find that the export (or import) volumes have a negative effect on the ratio between the median and the 10th percentile of the wage distribution and a positive effect on the ratio between the 90th percentile and the median of the wage distribution. As a result of these changes in trade-induced skill premium, the mean and the variance for the whole skill distribution increase. For Portugal, we find that the export (or import) volumes have a positive impact on our measures of wage gap and the effect is larger than the one estimated for Denmark. Despite the large estimates of tradeinduced skill premium for Portugal, their feedback effect on the first two moments of the skill distribution are negligible and not statistically significant. These results therefore show that the changes in trade-induced skill premium improve the overall level of skills but also make the population more diverse in terms of skills in economies such as Denmark while such effects are mitigated in frictional labor market settings in economies such as Portugal.

Our paper contributes to the existing literature on endogenous human capital investments (see for e.g., seminal work by Findlay and Kierzkowski, 1983) and more generally on how the skill distribution responds to trade shocks (Johnson and Stafford, 1999). The existing literature has either examined how trade affects the skill premium for a given skill distribution or how trade affects skill composition within an economy (for e.g., Costinot 
and Vogel, 2010; Egger and Kreickemeier, 2009; Yeaple, 2005; Maggi and Grossman, 2000). However, the past literature has not jointly studied these two issues, with a few recent exceptions. Blanchard and Willmann (2016) present a trade model that highlights how trade and educational institutions interact to determine individuals' skill acquisition decisions and the pattern of comparative advantage across countries. This framework is used to study how education or trade policies can be used to attenuate the "vanishing middle class" phenomenon recently observed in much of the industrialized world. Blanchard and Olney (2015) empirically show that educational attainment also responds to exogenously driven changes in the composition of country's exports, and thus they offer insight into how investment in human capital responds to changing patterns of production. Last, Atkin (2012) uses Mexican data to study the effects of export expansion on school attainment and finds that location and job characteristics are important determinants in understanding heterogeneous educational attainment responses to changes in trade policies.

Our work complements these existing studies in many ways. Unlike past work, we do not only focus on formal schooling and education as our skill measure. Instead, we rely on comprehensive definitions of skills and use other alternative definitions of skill measures. Moreover, comprehensiveness of our dataset also allows us to overcome some of the limitations that the past literature has encountered by using a database spanning several countries. For example, we study how trade affects the skill composition through changes in skill premium for the whole workforce employed in either the manufacturing or the service industry in contrast to Blanchard and Olney (2015), who focus on the skill acquisition of the young cohorts and the effects of exports within the manufacturing sectors. Finally our comparison of two extreme examples of labor market environment (Danish versus Portuguese), allows us to highlight the importance of labor market institutions in shaping the impact of trade on skill composition offering new insights on the role of frictions.

In the next section we present a conceptual framework that explains how changes in the skill premium affect the individual decisions to upgrade skills and their implications for the whole skill distribution. In Section 3 we present the institutional background for Denmark and Portugal. Data and summary statistics are then discussed in Section 4. Our empirical strategy is explained in Section 5. We presents our results in Section 6 and conclude in Section 7. Proofs, figures and tables are collected in appendices at the end. 


\section{Theoretical Intuition}

In this section, we present a simple 3-period model to highlight how skill premium affects skill distribution via its impact on individual's decision to upgrade skills first in a frictionless labor market and then in a frictional labor market.

\subsection{A Three-Period Skill Upgrading Example}

A country is populated by a continuum of heterogeneous agents with a unit mass. Each individual $i$ has a unique level of inherent ability $a_{i}$ where $0<\underline{a} \leq a_{i} \leq \bar{a}$ that does not change over one's life span. Ability, $a$, is distributed continuously with a cumulative distribution function denoted by $F(a)$ and corresponding density function denoted by $f(a)$.

Each individual lives for three periods where each period is of length one. Each period $j$, individual $i$ can decide to acquire higher skills or continue with the existing skills. We denote this decision using an indicator function $I_{i j}$ where $I_{i j}=1$ if individual $i$ decides to obtain the credit, otherwise $I_{i j}=0$. If $I_{i j}=0$ the individual will continue to earn wages $w\left(s_{i j}\right)$ based on her current skill level $s$. If $I_{i j}=1$, she will earn a higher wage $w\left(s_{i j}+\bar{e}\right)$ corresponding to her new skill level. However, acquiring skill requires a fixed amount of credits $\bar{e}$ and has an opportunity cost in terms of the wages not earned whilst the time spent for upgrading skills. We denote this opportunity cost by $t$ which increases with the required units of credits and a decreases with the innate ability of the individual. More succinctly $t_{i j}=\frac{\bar{e}}{a_{i}}$.

Each individual maximizes her life-time utility based on the consumption $c_{i j}$. We assume each individual can perfectly smooth their consumption over the life time, financed by her life-time income $W$, i.e., $W \equiv \sum_{j=1}^{3} w\left(s_{i j}\right) p_{j}\left(1-t_{i j}\right){ }^{2}$ We write individual $i$ 's skill choice problem as the following:

$$
V_{i}=\max _{I_{i j}} U\left(c_{i 1}\right)+\beta U\left(c_{i 2}\right)+\beta^{2} U\left(c_{i 3}\right)
$$

s.t.

$$
\sum_{j=1}^{3} c_{i j} p_{j}=\sum_{j=1}^{3} w\left(s_{i j}\right) p_{j}\left(1-t_{i j}\right), \quad s_{i j}=\underline{s}+\sum_{k=1}^{j-1} \bar{e} I_{i k}, \quad 0 \leq t_{i j}=I_{i j} \frac{\bar{e}}{a_{i}} \leq 1
$$

where $U\left(c_{i j}\right)$ denotes the utility from consumption, $p_{j}$ is price in each period, and $\underline{s}$ is the lowest skill level that every individual is born with in the first period and skill upgrading

\footnotetext{
${ }^{2}$ Relaxing this assumption requires a non-zero consumption $c_{i j}$ in each period. Qualitatively the analysis stays unchanged, therefore we make this assumption for simplicity so as to present closed form solutions for the follow up analysis.
} 
is simply an additive process to the previous skill level via earning fixed credit $\bar{e}$. We assume wages are a decreasing function of skills $\left(\frac{d w}{d s}>0\right)$ and concave $\left(\frac{d^{2} w}{d s^{2}}<0\right) .{ }^{3}$ Last, we assume that inflation is positive (i.e., $p_{1} \leq p_{2} \leq p_{3}$ ).

Under the assumptions discussed there exist ability thresholds, $\underline{a}<A_{1}<A_{2}<\bar{a}$ such that the thresholds are as follows:

$$
A_{1}=\frac{w(\underline{s}) p_{1} \bar{e}}{\left(p_{2}+p_{3}\right) \Delta_{1}}, \quad A_{2}=\frac{\left[w(\underline{s})+\Delta_{1}\right] p_{2} \bar{e}}{p_{3} \Delta_{2}}
$$

where $\Delta_{1} \equiv w(\underline{s}+\bar{e})-w(\underline{s})$ is the skill premium for the low-medium skill, $\Delta_{2} \equiv w(\underline{s}+2 \bar{e})-$ $w(\underline{s}+\bar{e})$ is the skill premium for medium-high-skill, and $\Delta_{1}>\Delta_{2}$.

If an individual's ability $a_{i} \leq A_{1}$, one's life-time utility is maximized when not upgrading skill at all. If an individual's ability $A_{1}<a_{i} \leq A_{2}$, one's life-time utility is maximized when upgrading skill once in the first period. If an individual's ability $a_{i}>A_{2}$, one's life-time utility is maximized when upgrading skill twice in the first two periods, respectively. We show detailed solutions in the Appendix. Without loss of generality, we assume that $A_{1}$ is below the medium ability while $A_{2}$ is above the medium ability. That is, $\bar{a}+\underline{a}<2 A_{2}$ and $\bar{a}+\underline{a}>2 A_{1}$.

Using this framework, it is straightforward to show that exogenous changes in skill premium denoted by $\Delta_{1}$ and $\Delta_{2}$ affect the threshold levels. In particular, increase in $\Delta_{1}$ pushes $A_{1}$ backwards $\left(\frac{\partial A_{1}}{\partial \Delta_{1}}<0\right)$ while $A_{2}$ forward $\left(\left.\frac{\partial A_{2}}{\partial \Delta_{1}}\right|_{\Delta_{2}} \mid\right)>0$. Increase in $\Delta_{2}$ does not affect $A_{1}$ but pushes $A_{2}$ backwards $\left(\left.\frac{\partial A_{2}}{\partial \Delta_{2}}\right|_{\Delta_{1}} \mid\right)<0$. These movements in thresholds in response to skill premium has implications for the overall skill distribution which are summarized below:

Proposition 1 Let $E(s)$ denotes the mean and $\operatorname{Var}(s)$ denotes the variation of skills. Suppose ability is uniformly distributed then:

- If $\left|\frac{\partial A_{1}}{\partial \Delta_{1}}\right|<\left|\frac{\partial A_{2}}{\partial \Delta_{1}}\right|_{\Delta_{2}} \mid$, an increase in $\Delta_{1}$ decreases the $E(s)$ and the $\operatorname{Var}(s)$.

- An increase in $\Delta_{2}$ increases the $E(s)$ and the $\operatorname{Var}(s)$.

Proof. See Appendix.

\footnotetext{
${ }^{3}$ These assumptions imply that there is always a positive return for upgrading skills in terms of wages and that the marginal benefit of upgrading skills diminishes. This is closer to what we observe in the data where the median annual earnings of full-time year-round wage and salary workers for ages 25 - 34 by educational attainment in USA in 2012 shows an approximate wage differential of $\$ 18,000$ for high school and college education versus $\$ 12,000$ for college and university education. See for example: http://nces.ed.gov/fastfacts/display.asp?id=77.
} 
The c.d.fs and the ability thresholds pin down the aggregate effects on the mean and the variance of skills in response to changes in skill premium. When $\Delta_{1}$ increases, the return to acquiring subsequent skills for the low ability individuals increases while the opportunity cost of acquiring subsequent skills for the medium ability individual increases. As a result, threshold $A_{1}$ and $A_{2}$ move further apart. More of the lower ability individuals who would not have upgraded skills ever will now upgrade skills once while less of the medium ability individuals who would have upgraded skills twice will now only upgrade skills once. Consequently, conditional on the relative movement of thresholds, the mean and variance will be affected. If $A_{2}$ moves less than $A_{1}$, then on average skills in the population are lower and the distribution of skills is more homogenous. In the case of an increase in $\Delta_{2}$ the return of acquiring skills twice increases and hence more of the medium skilled individuals would acquire skills twice resulting into a higher aggregate mean and more heterogeneous skill distribution.

\subsection{Labor Market Friction}

So far the labor market in the previous model is fully efficient in the sense that as long as the individual upgrades skills he/she will find a job that pays the skill-matching wage each period. Now we extend the model by assuming that the labor market is inefficient due to the presence of frictions, i.e., by assuming uncertainty in finding a job with skill-matching wage after skill upgrading. Such uncertainty could be justified on the basis of a very regulated labor market that limits turnover rates. We denote by $0<\theta \leq 1$ the probability of finding such a job with skill-matching wage each period, and by $(1-\theta)$ the probability of having a wage that corresponds to one level lower than the obtained skill. The smaller $\theta$ is, the more inefficient the labor market is. ${ }^{4}$

Under this additional assumption and assuming risk neutral agent, the new thresholds are now also a function of the labor market friction $\theta$ and the thresholds area as follows:

$$
A_{1}=\frac{w(\underline{s}) p_{1} \bar{e}}{\theta\left(p_{2}+p_{3}\right) \Delta_{1}}, \quad A_{2}=\frac{\left[w(\underline{s})+\theta \Delta_{1}\right] p_{2} \bar{e}}{p_{3}\left[\theta \Delta_{2}+(1-\theta) \Delta_{1}\right]}
$$

We restrict the range of $\theta$ such that the skill upgrading decision is monotonous. This is similar to assuming that $A_{1}<A_{2}$ to facilitate comparison across the two version of the model. ${ }^{5}$ Intuitively, labor market frictions affect the return and opportunity cost of skill

\footnotetext{
${ }^{4}$ See detailed mathematical setup in Appendix A.

${ }^{5}$ For $A_{1}<A_{2}$ to hold, $\theta$ needs to satisfy a certain condition. We prove that the condition is consistent with $0<\theta \leq 1$. See Appendix A for more details. Moreover, $\theta$ takes the range such that skill upgrading is monotonous. A person who finds skill upgrading once to be non-beneficial is not allowed to upgrade skills
} 
upgrading. The return in the case of frictionless market is higher but opportunity cost is lower for twice skill upgrading decision. Introducing such a reduced form of labor market frictions entails that when $\Delta_{1}$ increases, $A_{1}$ moves back but for smaller $\theta, A_{2}$ moves forward, while for larger $\theta, A_{2}$ moves backward, leaving the effect of $\Delta_{1}$ on the skill distribution ambiguous and conditional on $\theta$. When $\Delta_{2}$ increases, $A_{2}$ moves back hence the conclusion for skill distribution stays the same as the previous analysis except the effect is smaller when $\theta \neq 1$. We summarize our results as follows:

Proposition 2 Let $E(s)$ denotes the mean and $\operatorname{Var}(s)$ denotes the variation of skills. Suppose ability is uniformly distributed and $0<\theta<1$ then:

- An increase in $\Delta_{1}$ has ambiguous effect on the $E(s)$ and $\operatorname{Var}(s)$ of skills.

- An increase in $\Delta_{2}$ increases both the $E(s)$ and $\operatorname{Var}(s)$ of skills but by a smaller magnitude relative to when $\theta=1$.

Proof. See Appendix.

The first implication of the above proposition is that the aggregate distributional effects on the skills are less informative since the ultimate outcome does not only depend on the relative effect of $\Delta_{1}$ on $A_{1}$ and $A_{2}$ as in Proposition 1 but also on the size of $\theta$. In the case $\theta=1$, it is straightforward to see that $A_{2}$ increases in response to $\Delta_{1}$ but this is not the case when $\theta<1$. This is because the uncertainty in skill-wage matching simultaneously reduces the return and the opportunity cost of skill upgrading. It's then the relative reduction of these two components in the skill upgrading decision that determines whether $A_{2}$ increases, decreases or stays unchanged. ${ }^{6}$ It is therefore an empirical question to study the distributional effects of $\Delta_{1}$ on the skills in such an environment. The second implication of the proposition is that the effect of $\Delta_{2}$ on $A_{2}$ is always smaller in a frictional setting. In summary, in the presence of frictions in the labor market we are agnostic on the distributional effects of the skills when $\Delta_{1}$ changes but we are certain that the effects are expected to be much less pronounced when $\Delta_{2}$ changes.

twice either.

${ }^{6}$ Let $\bar{\epsilon}$ be a cut-off of $\theta$ such that when $\theta=\bar{\epsilon}$, reduction in return and cost of upgrading is equal and therefore $\left|\frac{\partial A_{2}}{\partial \Delta_{1}}\right|_{\Delta_{2}} \mid=0$; above $\bar{\epsilon}$, reduction in return is more than the reduction in the cost of upgrading and therefore $\left|\frac{\partial A_{2}}{\partial \Delta_{1}}\right|_{\Delta_{2}} \mid>0$, and below $\bar{\epsilon}$, reduction in cost is more than the reduction in the return of upgrading and therefore $\left|\frac{\partial A_{2}}{\partial \Delta_{1}}\right|_{\Delta_{2}} \mid<0$. 


\subsection{Connection with Empirical Analysis}

The set-up discussed in the previous section provide us with the theoretical underpinnings in order to study the effect of changes in skill premium on the skill distribution. The model provides two empirically testable implications. First for the frictionless market, (a) an increase in skill premium associated with the lower tail of the skill distribution has negative effects on both the mean and the variance of the entire workforce's skills, and (b) an increase in skill premium associated with the higher tail of the skill distribution has positive effects on the mean and the variance of the skill distribution. Second, for the frictional market (a) becomes ambiguous while (b) gets much less pronounced.

For the sake of providing a simple theory that focuses on the effects on the skill distribution triggered by changes in skill premium, we do not explicitly model the origins of the shocks to the skill premium. However in the empirical analysis, as mentioned in the introduction, we will focus on the skill premium changes which are induced via trade integration for a number of reasons. Trade integration has recently become one of the primary sources of changes in factor prices such as wages. This makes it relevant and interesting to study the effect of trade on the skill distribution not only from an economic but also from a policy point of view. Moreover, despite the recent discussions on the distributional effects of globalization, empirical analysis explicitly covering the feedback effect of trade on the skill composition has been so far inhibited by a number of empirical challenges, as highlighted in the introduction. Before moving to the empirical analysis, we first present the main insti-

tutional differences between Denmark and Portugal and then we describe the corresponding dataset for each country.

\section{Institutional Background}

In this section we explain the main features that define the trade patterns and the labor market in Denmark and Portugal.

\subsection{Trade patterns}

Both Denmark and Portugal are highly export oriented economies. The ratio of export volumes to GDP is in fact on a rising trend for both economies over the period analyzed in this study (see Figures 2 and 3).

Traditionally Danish and Portuguese trade has been limited to a few trading partners 
(in the 90's about 70\% of both Danish and Portuguese trade used to be with 10 countries, mostly EU members) but more recently both countries have also started to trade with emerging economies, such as the BRICs, east Asian countries and the formerly communist countries. Figures 4 and 5 show that export volumes for both countries have been on a very steep and positive trend when we look at destination markets outside the developed world, especially China. Thus despite the maturity of the Danish and Portuguese economies, the process of trade integration is still evolving for both countries for the period considered in our analysis. Another similarity across the two economies is in terms of the export composition, as both countries feature a large export shares within machinery equipment, textiles, food and transport (OECD, 2013, 2014).

\subsection{Labor Market and Educational Institution}

\subsubsection{The Danish Institutions}

Following from the long-standing tradition for open trade, globalization is generally seen as a positive force in Denmark. Indeed, the flexibility of the labor market means that Denmark is in a better position than many European countries to adapt to the changes in global market conditions brought about by the emergence of low-cost producer countries. Cornerstones of the Danish model are in fact a high level of labor mobility and generous social security schemes. The absence of severance pay lowers hiring and firing costs, reduces the matching frictions and facilitates firms to adjust the quality and size of their workforce. Moreover, although workers are not protected by stringent employment rules, they bear relatively low costs of changing employers and have easy access to unemployment or social assistance benefits and activation programs. In fact, Danish replacement ratios are among the most generous in the world.

Another key feature of the Danish labor market is related to the organization of the industrial relations. Historically all labor market issues have been settled in centralized negotiations between representatives for employers and employees without political interference. The social partners take full responsibility for wage bargaining and wage setting. They also make agreements on normal working hours and set rules together with the government for labor protection with respect to overtime and work environments. Moreover about $80 \%$ of all employees are members of a trade union in Denmark. The wage and working hours formation used to be highly centralized with biannual negotiations between trade union organization called Landsorganisationen i Danmark (LO) and Danish employers' federation (DA) in the private sector. Wage bargaining has recently become much more decentral- 
ized. Since the early 1980s, an increasing share of wage bargaining descended to the firm (individual employee) level, which increased the weight of employer and employee roles in the resulting internal firm wage structure. As found in Eriksson and Westergaard-Nielsen (2009), the within-firm wage variability in Denmark represents more than 80 percent of the total variability observed among all workers.

Finally the Danish government generally provides abundant subsidies for individuals skill upgrading and education. Formal schooling is largely provided for free at both the secondary and tertiary level and a monthly income transfer, i.e., statens uddannelsesstøtte, of about 700 dollars is provided to all Danish students during the entire course of their undergraduate and master studies. As a result of these policies, the educational level of the workforce is very high by international standards. In 2012 the population share having attained upper secondary education far exceeds the OECD average. The share having attained tertiary education is also above the OECD average (OECD, 2013, 2014). Moreover life-long learning opportunities are very accessible to those who are unemployed through the participation to active labor market policies.

As a result of these liberal educational policies combined with a very flexible labor market with limited matching frictions, the Danish workforce seems to be more equipped to adjust to skill premium changes induced by trade. Such responses therefore are more likely to be reflected in the changes in the skill distribution and composition.

\subsubsection{The Portuguese Institutions}

Contrary to the Danish labor market, the Portuguese one is, according to the OECD index of employment protection legislation strictness, one of the most rigid labor market in the world. As a result, Portugal features low labor turnover rates, very limited transitions between employment and unemployment, and very long unemployment spells (Blanchard and Portugal, 2001). Portugal is also characterized by a very regulated industrial relations system, where the wage setting consists of mandatory minimum wages and the widespread use of (centralized) collective wage bargaining at the sector level (Cardoso and Portugal, 2005). Roughly $90 \%$ of private sector jobs in Portugal are covered by sector-wide collective agreements negotiated by employer associations and trade unions (Addison et al., 2016). Wage clauses in collective agreements are typically updated every year and firm-level collective bargaining covers a low proportion of the workforce (less than 10\%).

The resulting collective agreements are both extensive and general. They are extensive

in the sense that they cover many categories of workers. They are also general as they only set minimum working conditions that become binding for the entire industry through 
administrative extension. The content of the agreements focus on wage floors for each category of workers, overtime pay, and the normal duration of work.

Under these conditions, the links between the wage growth defined by collective agreements and the actual economic conditions prevailing at the micro (firm) level can be very loose. Whatever the minimum wage level agreed upon for each category of workers at the collective bargaining table, firms are free to pay higher wages, and they often deviate from that benchmark, adjusting it to firm-specific conditions. As a consequence, employers have the possibility of tailoring remunerations to their prevailing economic circumstances (see for e.g., Cardoso and Portugal, 2005). On the other hand, this mechanism also discourages the entry of new firms and competition in product markets, as one way through which new firms can enter the market is to pay lower wages than incumbents for some time (OECD, 2014). Overall, Portugal is characterized by very high levels of wage inequality, which suggests that the wage setting is relatively unconstrained by institutional forces.

As far as education is concerned, Portugal is characterized by a workforce which is intensive in low skills. Over $60 \%$ of the 25-64 population has not yet completed upper secondary education, which is the third largest share in the OECD area (OECD, 2014). Despite the progress made in the reduction of early school leaving rate during the most recent years, the Portuguese indicators remain far above the targets set for Europe in 2020.

In general, tuition fees are higher than the minimum recommended in Portugal and student social support is very limited. Full time students enrolled in Bachelor or Master programs are charged an average tuition fee of around 950-1250 euros per academic year. For $\mathrm{PhD}$ degrees, the average tuition fee amount to approximately 3000 euros per academic year. As a result of this, Portugal is also one of the OECD countries where the association between students socio-economic background and their performance at school is higher, and children from highly educated parents are much more likely to participate in higher education than others, compared to other countries (OECD, 2014). Finally the Portuguese active labor market policies are characterized by low level of vocational training for the unemployed. Spending on active labor market policies (ALMPs) increased at the beginning of the crisis, peaked in 2008 at 0.63 per cent of GDP, but declined in the following years. The expenditure on ALMPs in Portugal is clearly below that of the Nordic countries which is above 1 per cent in Denmark from 2009 onwards (ILO, 2014).

A very rigid labor market combined with scarce public resources available for education and retraining are very likely to increase the frictions that hinder how the skill distribution adjusts to globalization. 


\section{Data}

\subsection{Data for Denmark}

Information about firms and workers is collected from several sources at the Danish official statistical institute (Denmark Statistics): the "Integrated Database for Labor Market Research" (IDA), the "Accounting Statistics Registers" (FirmStat), and the "Foreign Trade Statistics Register" (Udenrigshandelsstatistikken). From the population of all firms, we keep only firms which are comprised by all these registers over the period from 1993 to 2012. Moreover we only proceed with private companies. To avoid both self-employment, typical migrant businesses, we keep firms with more than 1 employee.

$I D A$ is a longitudinal employer-employee register, containing information on the age, gender, nationality, place of residence and work, education, labor market status, occupation, and wage of each individual aged 15-74 between 1980 and 2012. The information is updated once a year in week 48. Apart from deaths and permanent migration, there is no attrition in the data. The individual information in IDA is used to estimate our skill measure, as explained in the next section. This is then aggregated at one of the most detailed classification of the Danish Industrial activities (4-digit level) for the purpose of our empirical analysis. ${ }^{7}$ Individual-level variables in $I D A$ are also used to measure a number of workforce characteristics at the industry level: the share of workers with secondary and post-secondary education; the percentage of male employees; the average age and work experience. Because we can track people along the years, we can also establish the average tenure of all employees. In order to deal with outliers, top and bottom $1 \%$ of wage earners are excluded every year.

FirmStat is a firm registers which provide the annual value of capital stock, firm productivity and industry affiliation. ${ }^{8}$ This data captures some of the salient firm-level characteristics, which are then aggregated at 4-digit level classification of the Danish Industrial activities.

The "Foreign Trade Statistics Register" shows export sales and the number of exported products at the firm level. These data are available both at specific destinations and aggregated over all destinations. Exports are recorded in Danish kroner (DKK) according to the

\footnotetext{
${ }^{7}$ This industry classification corresponds to the 4-digit of the European classification, NACE rev.2. For more details on the Danish Industrial classification, please refer to http://dst.dk/da/Statistik/Publikationer/VisPub.aspx.

${ }^{8}$ The capital stock comprises the sum of the values (in Danish kroner) of land, buildings, machines, equipment and inventory. Firm productivity, which is the most important predictor of the export behavior of a firm in the recent trade theory, is calculated as turnover per employee in logarithmic scale (i.e., labor productivity). We deflate all monetary values using the World Bank's GDP deflator with 2005 as the base year.
} 
8-digit Combined Nomenclature as long as the transaction is at least worth 7500 DKK or involves goods whose weight is at least $1000 \mathrm{~kg} .{ }^{9}$ To construct our instruments, as explained in the next section, we aggregate these flows to the 6 digit Harmonized System (HS) in order to improve the merge to the COMTRADE data. Moreover, as we did for all the other variables included in the empirical analysis, we calculate export sales at 4-digit level of the industry classification.

\subsection{Data for Portugal}

Our analysis for Portugal is based on data from Quadros de Pessoal (QP). QP is, by construction, a longitudinal matched employer-employee dataset. This is an annual mandatory employment survey collected by the Portuguese ministry of employment covering all firms (and each of its establishments) with at least one wage earner. Every year firms with paid employees are legally obliged to fill in a standardized questionnaire, reporting information at three levels: the establishment (location, industry, and employment), the firm (location, industry, employment, sales, ownership, and legal setting), and each of its workers (gender, age, education, skills, occupational category, employment status, earnings, citizenship, and tenure). Self-employed individuals without employment are, hence, not covered in QP.

The information on earnings is very detailed, precise, and complete. It includes the base wage (gross pay for normal hours of work), regular benefits, and overtime pay. Because the information on earnings is reported by the employer, it is unlikely to be subject to measurement error relative to worker-provided earnings data. The fact that the information contained in the QP survey must by law be available in a public space at the establishment further reinforces our trust in the information. We construct our monthly wage measure by taking into consideration both workers base salary and any regular earning supplements. In order to deal with outliers, top and bottom $1 \%$ wage earners are excluded every year.

Our analysis is based on all firms comprised by the registers over the period 1993-2011. ${ }^{10}$ Individual-level data files were used to estimate our skill measures and to characterize the workforce (share of workers with secondary and tertiary education, the percentage of male employees, average age, and work experience). Workforce and firm characteristics (namely labor productivity) were then aggregated at the industry-level (3-digit). ${ }^{11}$ Trade information

\footnotetext{
${ }^{9} 7500$ DKK are about 1000 euros at the time of writing. Since the introduction of the euro currency, the Danish Central Bank has adopted a fixed exchange rate policy vis-a-vis the euro.

${ }^{10}$ The year 2001 is missing as information for this year was not collected by Statistics Portugal.

${ }^{11}$ In order to perform our empirical analysis over the whole period (1993-2011), we standardize all industries according to the earlier version of NACE rev. 1.1, which is more aggregate than later versions (NACE rev 2.). This gives around 80 (3-digit) industries in total every year.
} 
at the industry-level was obtained from Statistics Portugal and merged with QP data. To construct our instruments, we used trade information from COMTRADE at the productlevel. The link between each 3-digit industry, the respective 4-digit products exported (imported), and the country of destination (origin) was also obtained from Statistics Portugal.

\subsection{Descriptive statistics}

Table 1 groups the descriptive statistics of all our main variables for both Denmark and Portugal. Denmark features on average a larger (smaller) ratio of the 50th (90th) to the 10th (50th) percentiles of the earnings distribution compared to Portugal. As we will explain in the next session, those wage ratios will be interpreted in the empirical analysis as proxies for the low-medium skill and the medium-high skill premium. The first panel of Figures 6 and 7 describe their over time patterns: for both Denmark and Portugal the mediumhigh skill premium is on a positive trend, whereas the development of the low-medium skill premium looks very different across the two countries. Whereas for Portugal the wage ratio, $\frac{w 50}{w 10}$, steadily increases up to 2008 , the same ratio is on a flat, slightly negative, trend for Denmark, even if its pattern seems to be very noisier than its Portuguese counterpart especially in the last part of the period observed.

As far as the skill variable is concerned, we will proceed with a number of alternative measures. The first three definitions represents our main measures and they are all estimated from the additive "two-way" worker-firm effects model (Abowd et al., 1999), as explained in the next section. These variables represent a good proxy for all the skills acquired formally at school and informally on the labor market. As a robustness check, we will also provide the results on the basis of two other measures of skills. The first one is based on years of education (definition 4) whereas the second one is a multidimensional index drawn from Portela (2001) (definition 5). According to definitions 4 and 5, Denmark has on average a higher endowment of skills compared to Portugal. ${ }^{12}$ The second panel of Figures 6 and 7 report the overtime developments of the first two moments of skills measured by using the fourth definition of skills, which is the only one directly comparable across the two countries. These figures show that both the average and standard deviation of skills has been steeply increasing for Denmark. The same holds true for Portugal, even though the positive trend on the mean and the standard deviation are much less pronounced compared to its Danish counterparts. In the empirical analysis we will analyze how much of these developments

\footnotetext{
${ }^{12}$ Direct comparison of definitions 1,2 and 3 is not feasible given that they originate from different measures of wages, as it will be clearer in the next section.
} 
in the skill distribution are specifically explained by the skill premium changes induced by overtime increases in export or import activity.

Our trade measures will be mainly based on the export volumes, although in a robustness check we also look at the import volumes. Denmark features higher volumes of exports, overall and by destination, and of imports compared to Portugal. Finally we also consider a number of industry-level characteristics, as control variables. Specifically we include in the analysis a measure of capital intensity ${ }^{13}$ and productivity as proxies for technology at the industry level and a whole host of workforce composition characteristics, as indirect measures of the quality of the labor force in each industry. Compared to the Portuguese one, the Danish case seems to be characterized by higher level of productivity, a larger fraction of workers with either secondary or tertiary education and shorter employees' tenure on average. Similar statistics across the two countries are recorded for the share of male employees and for employees' average age and work experience.

\section{$5 \quad$ Methodology}

We organize this section into two parts. The first part describes how we calculate our main measure of skills. The second part then discuss how we empirically test the main theoretical predictions on the first two moments of the skill distribution.

\subsection{Skill measure}

Our point of departure for the measurement of the main skill variables is the following gender-specific wage equation à la Abowd et al. (1999):

$$
\ln w_{i j t}=\alpha_{i}+\psi_{j(i, t)}+\beta_{1} X_{i t},+\varepsilon_{i j t}
$$

where $w_{i j t}$ is the gross wage earned by individual $i$ in firm $j$ in year $t .^{14}$

$X_{i t}$ is a vector of individual specific controls that changes overtime. We include in

\footnotetext{
${ }^{13}$ Unfortunately capital intensity is not available for Portugal. We partially compensate for this important missing information by including 2-digit industry fixed effects in our regression models.

${ }^{14}$ For Denmark the wage variable is represented by the annual gross wages. The annual wages are in real terms and adjusted for possible unemployment spells during the year. In additional analysis for Denmark not reported in the paper, we get similar results by focusing on wages corresponding to the November spell only. In this case, for those employees who work for fewer than 365 days at the firm corresponding to the November spell (around 35 percent), we annualize their wages by using information on the duration of the same spell. For Portugal we use wages for the month of October of each year.
} 
$X$ the workers' education interacted with year dummies. In addition, we also control for other factors that might affect wages such as age, experience and tenure and their squares. The parameters $\alpha_{i}$ and $\psi_{j(i, t)}$ are respectively the individual and firm specific fixed effects. We estimate this additive "two-way" worker-firm effects model by using the methodology developed in (Abowd et al., 1999) for each gender separately. The results from this additive fixed effects model are reported in Table 2 and look fairly comparable in terms of signs estimated on the main coefficients across the two countries.

This methodology is based on the identification of connected sets of firms which are firms that have movers in common. Due to the high mobility that characterizes the Danish labor market and the relatively long time period considered, the largest connected set contains more than $99 \%$ of the workers and firms in the sample. Moreover this methodology relies on the assumption of "conditional exogenous mobility". As suggested in Card et al. (2013) for Germany and in Card et al. (2016) for Portugal, we empirically assess its plausibility for the Danish case by considering all possible cases in which this assumption may be violated.

First, we consider the case of sorting based on the idiosyncratic employer-employee match component of wages. This type of sorting is problematic because workers are paid differently at each firm depending on the match component. Absent any match effect, the average wage gains and losses from moving between high and low wage firms are expected to be symmetric. The existence of match effects however, will tend to offset the losses associated with moving to a low wage firm. If all transitions are voluntary and selection is based only on the match component, movers would experience no wage losses.

Following Card et al. (2013), we construct mean log co-workers wages for each person in each year. We assign each worker to a quartile of the co-workers wage distribution. We do it separately for men and women. In each year we then derive average wages of movers by quartiles. Movers are defined as those who move from one firm to the other and who can be observed for two consecutive years in both the sending and the receiving firm. Figure 8 and 9 present the results of this analysis using data for male and female workers in Denmark. The corresponding figures for Portugal can be found in Card et al. (2016). For clarity, only the wage profiles of workers who move from jobs in quartile 1 and quartile 4 are shown in the figures. Similar to Card et al. (2016) among others, we find rather symmetric wage losses and wage gains for workers moving from high to low paying firms and the opposite. We do not find big wage changes for workers moving across firms paying similar wages. This suggests that the sorting based on a match component is likely to play a minor role in our setting.

A second case in which the exogenous conditional mobility is violated is when mobility is 
related to unobserved and temporary firm specific shocks. In this case for example, workers might be more likely to leave firms that experience negative shocks so as to join those firms which experience positive shocks. As in other studies however, we fail to find evidence in the data of particular dips in the wages of leavers or exceptional growth in the wages of joiners (Figures 8 and 9).

Finally a third problematic case might arise if mobility is related to unobserved temporary individual shocks. This is the case for example, if workers who are performing well earn higher wages in the sending firm and they move to high paying firms and those who are performing worse experience wage cuts prior to moving to low paying firms. ${ }^{15}$ Under this hypothesis, we would observe different trends prior to moving for workers who end up in high versus low paying firms. We do not find particularly different pre-trends for workers moving to high versus low paying firms (Figures 8 and 9), suggesting that temporary shocks have a a small effect on mobility in our sample. All in all these findings seem to support the exogeneity condition discussed above.

In the main analysis, our skill variable is then constructed for each male and female worker from equation by considering the systematic part only, i.e. $\beta_{1} X_{i t}$. In an alternative specification we also calculate the skill measure by excluding either age or both age and tenure from the systematic part, as those variables may be mechanically related to wages and consequently may not reflect any upgrading of the skill endowments. We then aggregate all these skill measures at the industry level.

\subsection{Skill premium and skill distribution}

We now present the empirical set-up used in order to test the main predictions described in the theory section. Specifically we present the regression models in which we investigate how the trade induced changes in the skill premium affect not only the average skill levels but also the skill variance in the economy. The theoretical underpinnings highlighted in the previous section suggest that the effects on the mean and variance of skills depends on the type of skill premium considered. To explore this hypothesis, we first estimate the following equation:

$$
\text { skill_premium }_{j t}=\alpha+\beta^{\prime} X_{j t}+\gamma \text { trade }_{j t}+\delta_{j}+\delta_{t}+\epsilon_{j t}
$$

\footnotetext{
${ }^{15}$ This might for example also be the case when ability is slowly revealed overtime.
} 
where the dependent variable is measured as the ratio between the 90th and the 50th percentile of wage distribution $\left(\frac{w 90}{w 50}\right)$ of industry $j$ at time $t$, as a proxy for the medium-high skill premium, i.e. $\Delta_{2}$ in our model. Or alternatively as the ratio between the 50th and the 10th percentile of wage distribution $\left(\frac{w 50}{w 10}\right)$ of the industry level $j$ at time $t$, as a proxy for the low-medium skill premium, i.e. $\Delta_{1}$ in our model. We measure the variable trade $e_{j t}$ with the industry-level export volumes aggregated over all destinations. ${ }^{16}$ The vector $X_{j t}$ includes a set of confounding factors that may effect the skill premium such as productivity, capital intensity and workforce composition characteristics (such as the share of tertiary educated employees and male workers) for each industry $j$. The specification of equation (1) is completed with a full set of industry fixed effects denoted by $\delta_{j}$ and time fixed effects denoted by $\delta_{t}$.

To obtain an unbiased estimate of the parameter $\gamma$ in equation (4), we instrument the export volume variables with exogenous trade shocks at the industry level. Exogenous trade shocks are constructed using changes in the world import demand for each product by using the COMTRADE data following the IV approach employed in Hummels et al. (2014) and aggregated at the industry level. We denote our instrument variable by $m_{j t}$. More specifically, the instrument is constructed jointly for the total exports of Denmark and Portugal as follows:

$$
m_{j t}=\sum_{c=1}^{C} \sum_{p=1}^{P} \frac{e x p_{j c p \_b a s e y e a r}}{e x p_{j \_ \text {baseyear }}} I_{c p t}
$$

where $I_{c p t}$ is each country c's total purchases of product $p$ from the world market (less purchases from Denmark) at time $t$ (Hummels et al., 2014). The variable $\exp _{\text {jcp_baseyear }}$ are the industry $j$ 's export volumes of product $p$ to the destination $c$ at the base year (which is

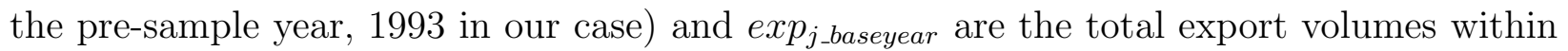
each industry $j$. These shocks are external to Denmark and they vary across destinations, countries and products. The weights $\frac{e x p_{\text {jcp_baseyear }}}{e x p_{j \_ \text {baseyear }}}$ are the base-year (1993) export shares, which are industry-specific and should be exogenous to over time changes in the level or the type of technology, which may potentially affect both exports and the skill premium at the industry level.

\footnotetext{
${ }^{16}$ In an alternative specification implemented in the robustness checks section, we either measure the industry-level export volumes separately for "north" and "south" destinations or we use import volumes over all destinations are as a trade variable. "North" includes countries of North America, Oceania, Western and Southern Europe (excluding the new EU members of the 2004 enlargement). All the other countries in the world are considered under the category of "South".
} 
Since our theoretical model does not explicitly model the causes for skill premium changes, we are agnostic about the sign of $\gamma$. However, following the standard practice in this field (Costinot and Vogel, 2010), we can predict the separate effects of the export volumes to north and south on the skill premium. Then the aggregate effect of all export volumes is going to be a combination of the effects stemming from the two types of trade.

Specifically, we expect the export volumes from Denmark or Portugal to south to have a negative impact on the wage differentials corresponding to lower tail of the skill distribution and a positive impact on the wage differentials corresponding to the higher tail of the skill distribution. South typically has lower endowment of skills, i.e. is less skill abundant, it is therefore very likely that the skill premium for the upper tail of distribution, i.e., $\frac{w 90}{w 50}$, increases on average. This is due to the fact that: 1) the employment share in tasks with high skill intensities increases within the economy ; 2) high skill workers have a comparative advantage in tasks with high-skill intensities (they experience a higher wage increase than the other workers). The opposite is expected to happen to the wage of those skills which are comparable to those prevailing in south, i.e., to $\frac{w 50}{w 10}$.

The export volumes to north are expected to have an ambiguous effect on our measures of wage differentials. The sign on the coefficient $\gamma$ estimated on $\frac{w 90}{w 50}$ or $\frac{w 50}{w 10}$ is therefore not clear cut a-priori, as it depends on the difference in skill diversity between Denmark or Portugal and their trading partners on average. If the distribution of skills in Denmark or Portugal is on average more compressed and more homogeneous than the one prevailing in north, then the $\gamma$ estimated on $\frac{w 90}{w 50}\left(\frac{w 50}{w 10}\right)$ is expected to be negative (positive) and we should observe wage convergence. This occurs because exporting industries tend to specialize in activities that require workers with similar skills to work together, i.e. technologies demanding skill homogeneity and complementarity. The opposite holds if the distribution of skills in Denmark or Portugal is on average more heterogeneous than the one prevailing in north, i.e., we should observe wage polarization, as industries specialize in production processes requiring skill diversity, i.e. industries intensify the adoption of sub-modular technologies. As in our baseline specification, we mainly focus on total export volumes aggregated over all destinations, we will estimate a coefficient $\gamma$ that represents a combination of all the mechanisms involved in the exporting activities to both north and south.

Once we have estimated equation 4 , we then predict the skill premium at the industry level (i.e. the exogenous component due to trade shocks) and we plug the latter in the following regressions in order to test how overtime changes in the skill premium induced by trade subsequently affect not only the average skills denoted by $s k \bar{i} l_{j t}$ but also skill diversity, which is measured by the standard deviation of skills and denoted by $\sigma\left(s k i l l_{j t}\right)$ : 


$$
\begin{aligned}
& \text { skill }_{j t}=\alpha+\beta^{\prime} X_{j t-1}+\gamma_{a v e}^{\prime} \text { skill_prêmium }_{j t-1}+\delta_{j}+\delta_{t}+\epsilon_{j t} \\
& \sigma\left(\text { skill }_{j t}\right)=\alpha+\beta^{\prime} X_{j t-1}+\gamma_{d i s p}^{\prime} \text { skill_premium } \hat{j}_{j t-1}+\delta_{j}+\delta_{t}+\epsilon_{j t}
\end{aligned}
$$

where the explanatory variable (skill_premium $\left.{ }_{j t-1}\right)$ is calculated as $\left(\gamma^{i v}\right.$ trade $\left._{j t-1}\right)$, with $\gamma^{i v}$ estimated from equation 4 using our IV approach. ${ }^{17}$ The first two moments of the skill distribution at the industrial level $j$ will be based on the skill variable, as described in the previous sections.

\section{$6 \quad$ Results}

Our main results for Denmark can be summarized as follows. On the one hand, the skill premium associated to the lower (upper) tail of the distribution are negatively (positively) affected by trade activity. On the other, an increase in these trade-induced skill premium has asymmetric effects on the skill distribution: (1) an increase in the skill premium associated with the lower tail of the distribution results in lower average skills and in a more homogenous skill distribution, (2) an increase in the skill premium associated with the upper tail of the distribution increases the overall average skills and it also makes the skill distribution more heterogenous. Interpreting the results from the first and second steps jointly together allows us to conclude that trade integration increases the overall mean and standard deviation of the skill distribution in Denmark.

For Portugal, we find instead that trade has significant and positive effects on both measures of skill premium. However, these trade-induced skill premium changes don't seem to influence the overall skill distribution, i.e., both the average level of skills as well as the overall skill dispersion remain largely unaffected. The stark difference in the distributional effects across the two countries further highlights that Denmark's "flexicurity" model allows its labor market to smoothly adjust to changes stemming from globalization and trade integration. Portugal on the other hand is characterized by extensive employment protection legislations and limited support for skill upgrading opportunities both of which hinder the ability of the workforce to respond to trade shocks efficiently. Therefore among other differences across the two countries labor market flexibility may play an important role in explaining such heterogenous responses to trade-induced skill premium changes. In the following section, we

\footnotetext{
${ }^{17}$ Due to collinearity issues, we have to include the predicted changes in the skill premium, skill_premium $_{j t-1}$, separately for $\frac{w 90}{w 50}$ and $\frac{w 50}{w 10}$ in equations and. We further investigate whether this omission leads to biased coefficients in one of the last robustness exercises reported in the results section.
} 
first discuss in details the baseline results and then present a whole host of robustness checks that allows us to corroborate our main findings.

\subsection{Main Results}

The estimated coefficients of the first and second steps of our econometric specification are presented in Table 3. In the first stage we tease out the impact of the trade on our two measures of skill premium, by instrumenting the export volumes with shocks to world import demand, as in Hummels et al. (2014). For Denmark, we find that an increase in trade volumes induce wages for high skilled workers to diverge and wages for low skilled workers to converge. Specifically, a standard deviation increase in the export volumes causes approximately 1 (2) percent increase (decrease) in $\frac{w 90}{w 50}\left(\frac{w 50}{w 10}\right){ }^{18}$ For Portugal both measures of wage gaps are positively affected by an increase in the trade volumes and the magnitudes involved are larger than the ones estimated for the Danish case. A standard deviation increase in the export volumes implies approximately $8.5(4.3)$ percent increase in $\frac{w 90}{w 50}\left(\frac{w 50}{w 10}\right){ }^{19}$

In the second step we estimate the impact of an increase in the predicted skill premium induced by the log of export volumes on the first two moments of the overall skill distribution, by using the first definition of skills. The effects of trade-induced skill premium on the overall skill distribution are significant for the Danish case but not for Portugal. For Denmark, a standard deviation increase in the predicted skill premium $\frac{w 90}{w 50}\left(\frac{w 50}{w 10}\right)$ leads to a 9 percent increase (6.5 percent decrease) in the overall mean of skills. Moreover, a standard deviation increase in the predicted skill premium $\frac{w 90}{w 50}\left(\frac{w 50}{w 10}\right)$ leads to a 10 percent increase (marginally insignificant) in the overall standard deviation of skills. Combining the results from the first and the second stage reveals that the proportion of those individuals with medium skill upgrading their skills to the next level is higher as a result of the decrease in the low-medium skill premium and of the increase in medium-high skill premium. As a result, the Danish skill distribution shifts to the right and becomes more dispersed.

For Portugal, none of the effects estimated for $\frac{w \hat{5} 0}{w 10}$ and $\frac{w \hat{9} 0}{w 50}$ are statistically significant. Moreover, despite the statistically and economically significant impact of export volumes on both measures of skill premium, these trade-induced changes in the skill premium fail to affect the overall skill distribution. ${ }^{20}$

\footnotetext{
${ }^{18}$ These figures are obtained by using the averages of $\frac{w 90}{w 50}$ and $\frac{w 50}{w 10}$ and the standard deviation of the $\log$ of the export volumes from Table 1.

${ }^{19}$ These figures are obtained by using the averages of $\frac{w 90}{w 50}$ and $\frac{w 50}{w 10}$ and the standard deviation of the $\log$ of the export volumes from Table 1.

${ }^{20}$ The large sizes involved in the skill premium changes estimated for Portugal also allow us to dismiss the surmise that for these distributional effects to show up it is necessary that the skill premium changes
} 
These results are intuitive and in line with our theoretical predictions. Contrary to an economic setting where the responses to changes in skill premium are hindered by a number of domestic frictions (such as high educational costs and a very restrictive employment protection legislation), flexible labor markets (such as the Danish one) are expected to show significant responses to trade in terms of the skill distribution.

\subsection{Robustness checks}

\subsubsection{Alternative skill definitions}

In this section, as a part of our robustness checks, we first evaluate the variations in the coefficients estimated for the second step that result when the skill variable is calculated in various ways. First, we rule out age (in skill definition 2) or both age and tenure (in skill definition 3) from the skill measure based on the systematic component of wage regression . This is done to take into account those cases in which age or tenure in the wage regressions don't exactly reflect the upgrading of the skills acquired on the labor market but rather a mechanical improvement of wages related to seniority. Buhai et al. (2014) document for example that there are significant returns to seniority in Denmark and Portugal and the effects are twice as large in Portugal relative to Denmark. The results obtained from skill definitions 2 and 3 are reported in the first and second sub-panels of Table 4 for Denmark and of Table 5 for Portugal. Reassuringly, these findings are fairly comparable to the main results reported in Table 3, especially in terms of the impact on the overall average skills.

Given that the first three definitions of skills based on the AKM model requires the rather restrictive assumption of "conditional exogenous mobility", we also present the results by using two additional alternative measures of skills, that are perhaps less controversial within the labor economics literature and also allow a direct comparison with the other existing studies. The first one simply relies on the workers' years of education as in Blanchard and Olney (2015) and as such it does not require any particular assumption. However the main limitations of this measure is that it only captures those skills acquired through formal schooling and it does adjust for the quality of skills. This corresponds to skill definition 4 in Tables 4 and 5. The second one is drawn from Portela (2001) and it is a multidimensional index of workers' human capital. Specifically, this index is constructed at the worker level by combining information on employees' years of education, work experience and unobserved ability estimated from a standard mincer equation with individual fixed effects, as follows:

exceed a certain threshold. We observe in fact that skill premium changes of smaller can indeed affect the skill distribution, as it is shown in the Danish case. 


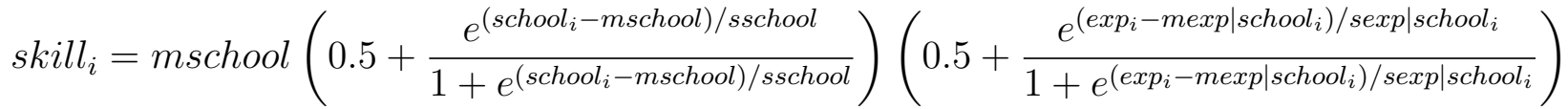

$$
\begin{aligned}
& \left(0.5+\frac{e^{\left(\text {effect }_{i}-\text { meffect } \mid \text { school }_{i} \text { exp }_{i}\right) / \text { seffect } \text { school }_{i} \text { exp }_{i}}}{1+e^{\left(\text {effect }_{i}-\text { meffect } \mid \text { school }_{i} \exp _{i}\right) / \text { seffect } \text { school }_{i} \text { exp }_{i}}}\right)
\end{aligned}
$$

where $m(s)$ school, $m(s) \exp$ and $m(s)$ effect are respectively the mean (standard deviation) of years of education, work experience and unobserved ability in the economy, whereas school $_{i}, \exp _{i}$ and effect $i$ are the same variables measured for each worker $i$. The skill measure skill $_{i}$ assumes that all the workers entered the labor market with the average education in the economy, mschool. This average is then adjusted to take into account worker $i$ 's relative position in the distribution of schooling. This correction is computed by considering the cumulative logistic distribution. The next step consists of adjusting the skill measure for the work experience by considering it as complementary to education, i.e. by comparing only the experience of individuals with the same years of education. This second correction reflects the fact that individuals with the same years of education may have different skills as a result of a different work experience. After adjusting the years of education for the work experience, we use a similar procedure to correct for the dispersion of unobserved ability within those workers who have the same schooling and experience. This allows us to calculate the measure of skills of worker $i$, skill $i$, which corresponds to skill definition 5 in Tables 4 and 5 .

By using these two additional alternative methods to calculate the first two moments of the skill distribution for the whole economy, we find that the effects of the skill premium changes induced by trade on the skill distribution are fairly similar in sign with those reported in Table 3 for Denmark. However the magnitudes involved in these definitions are smaller (larger) compared to the baseline definition, if we look at the impact of $\frac{w 90}{w 50}$ on the mean (the standard deviation) of skills for the whole economy in Denmark. If we take the skill definition 4, for example, a standard deviation increase in the predicted skill premium $\frac{w 90}{w 50}$ leads to nearly a 4 (30) percent increase in the overall mean (standard deviation) of skills. As in the baseline results, none of the effects on the skill distribution is precisely estimated for Portugal.

\subsubsection{Alternative measures of trade activity}

In this section, we proceed by assessing the sensitivity of our main results to alternative measures of trade activity. First, we investigate whether the effects triggered by trade on 
our measures of skill premium are heterogeneous across destination markets. We therefore distinguish between the export volumes directed to the developed economies (north) and those targeting the developing countries (south). Table 6 includes the results separately for north and south obtained for the first and the second step of our empirical strategy. The first sub-panel of Table 6 considers the Danish case and it reveals that most of the effects estimated on the export volumes on our measures of wage differentials are driven by the trade with south, as the impact of trade with north is never statistically significant. The fact that $\frac{w 90}{w 50}$ increases with the exports to south and that $\frac{w 50}{w 10}$ is negatively affected by the same type of trade is consistent with the Samuelson-Stolper hypothesis (Costinot and Vogel, 2010). The combined effects of trade with south on the skill distribution are fairly consistent with the baseline results, i.e. the changes in the skill premium triggered by trade with south induces a shift of the overall mean to the right and an increase in skill dispersion in Denmark. The second sub-panel of Table 6 presents the results for Portugal and shows that for Portugal there is not enough variation to separately identify the impact of trade with north and south on our measures of skill premium. ${ }^{21}$

In the next sensitivity exercise, we replace the export with the import volumes as a measure of trade activity. For these estimations, we re-calculate our instrument by looking at the changes in the world export supply for each product (minus Denmark's or Portugal's export volumes) gathered by COMTRADE data. Our assumption is that a measure based on the rest of the world's export supply can be safely assumed to be exogenous to the Danish or Portuguese import activities. The results based on this alternative measure of trade are reported in Table 7. The estimates for both Denmark and Portugal are very similar to our main findings, i.e. import volumes affects our measures of skill premium in both economies but the impact on the first two moments of the skill distribution is statistically significant only in the Danish case. A standard deviation increase in the predicted skill premium $\frac{w 90}{w 50}\left(\frac{w 50}{w 10}\right)$ leads to a 5 percent increase (3.5 percent decrease) in the overall mean of skills. Moreover, a standard deviation increase in the predicted skill premium $\frac{w 90}{w 50}\left(\frac{w 50}{w 10}\right)$ leads to a 12 percent increase (9 percent decrease) in the overall standard deviation of skills. The impact of the import volumes on the skill distribution is therefore fairly comparable to the one estimated when trade activity is proxied by the export volumes instead.

We finally investigate whether the impact of trade on the skill distribution depends on type of product exported. Blanchard and Olney (2015) show that the composition of trade plays a crucial role in affecting the incentives for the acquisition of education. Growth in less skill-intensive exports depresses average educational attainment while growth in skill-

\footnotetext{
${ }^{21}$ The second stage cannot be estimated as we only include those skill premium changes that are significantly related to trade activities in the first stage.
} 
intensive exports increases schooling. However, the estimated coefficient on the overall aggregate measure of exports may hide these opposite effects on the acquisitions of skills. We therefore construct the export volumes variable separately for high or low skill intensive industries. To do so, we draw a distinction between industries with R\&D expenditures above the overall economy average (regarded as high-tech industries) and those with below average R\&D expenditures (defined as low-tech industries). Information on R\&D expenditures at the 3-digit NACE industry level is retrieved from the OECD database for both Denmark and Portugal. Table 8 and Table 9 respectively present the results for trade activity separately for high- and low-tech industries.

Unlike the evidence reported in Blanchard and Olney (2015), we don't find substantially different patterns across these two groups of sectors. Our results are fairly consistent with the baseline evidence reported in Table 3. On the one hand for the Danish case, the overall mean and standard deviation of skills is positively (negatively) affected by predicted skill premium $\frac{w 90}{w 50}\left(\frac{w 50}{w 10}\right)$ in both types of sectors. However the magnitudes involved are larger when we focus on the high-tech industries compared to the main analysis. A standard deviation increase in the predicted skill premium $\frac{w 90}{w 50}\left(\frac{w 50}{w 10}\right)$ leads to a 20 percent increase $(5$ percent decrease) in the overall mean of skills. Moreover, a standard deviation increase in the predicted skill premium $\frac{w 90}{w 50}\left(\frac{w 50}{w 10}\right)$ leads to a 33 percent increase ( 8 percent decrease) in the overall standard deviation of skills.

On the other hand for Portugal, while the impact of the predicted skill premium on the standard deviation of skills is not significant as in the main analysis, the same impact on the average skills is negative and significant, at least in the high-tech sectors. These results alone may be interpreted as suggestive evidence of the skill convergence hypothesis resulting from a specialization in technologies that require skill homogeneity, which may depress average skill in the tech-industry (Costinot and Vogel, 2010). However the magnitude involved in the estimated effect on the average skills is very limited. Moreover this interpretation is not supported by the result on skill diversity and it's at odds with our theoretical propositions on the role of frictions in the labor market.

\subsubsection{Alternative IV strategies}

One of the main assumptions behind our identification strategy is that changes in non-Danish (or non-Portuguese) imports from non-Danish (or non-Portuguese) markets are orthogonal to industry-specific shocks occurring in either Denmark or Portugal. We deem this assumption plausible for our two economies given that both Denmark and Portugal are small countries of less than 10 million people and represents a small share of trade, both in the aggregate and for 
individual partners and products. Moreover all of our specifications include year and industry dummies beside time-varying controls. However in order to deal with correlation between the instrument and the residual in the wage differentials in equation 4 , we exclude those countries which are the most similar to either Denmark or Portugal in terms of business cycle. We therefore rule out from equation 5 Germany, Sweden and USA in the Danish application and Spain, France and Germany for Portugal. This is done in order to address the concern that higher imports from these markets may be correlated with the error term of equation 4, if they are triggered by demand shocks correlated with those occurring in Denmark and Portugal. The results obtained from this alternative specification of the instrument are reported in Table 10 and fairly comparable to those obtained in the baseline analysis.

Next, as an additional check, we re-estimate our baseline specification excluding industries in which demand or technology shocks are more likely to be correlated across countries. Following Colantone et al. (2015), these are: manufacture of coke, refined petroleum products and nuclear fuel (NACE 23; manufacture of rubber and plastic products (NACE 25); manufacture of radio, television and communication equipment and apparatus (NACE 32); air transport (NACE 62); post and telecommunications. Alternatively, we exclude the seven industries originally identified by Autor et al. (2013) as having experienced substantial fluctuations over the sample period across countries, due to technological innovations, housing booms, and the rapid growth of emerging economies. These industries are: manufacture of textiles (NACE 17); manufacture of wearing apparel; dressing an dyeing of fur (NACE 18); tanning and dressing of leather; manufacture of luggage, handbags, saddlery, harness and footwear (NACE 19); manufacture of other non-metallic mineral products (NACE 26); manufacture of basic metals (NACE 27); manufacture of fabricated metal products, except machinery and equipment (NACE 28); manufacture of office machinery and computers (NACE 30). The evidence obtained from both exclusions is reported in Tables 11 and 12 and confirms the robustness of our main results to the main concerns regarding our identification strategy.

\subsubsection{Additional checks}

Our results on the skill distribution for Portugal are never precisely estimated. It could be argued that this is due to the fact that we only observe a more aggregated classification of the Portuguese industries. We assess whether this is the case by running both the first and the second stage for Denmark on a more aggregate level, by using 3-digit level of industry classification (instead of a 4-digit classification). The results reported in Table 13 allows us to dismiss this concern, as the estimated results for Denmark's skill distribution are fairly 
comparable to the main results obtained from more disaggregated data.

The fact that we have to include the predicted changes in the skill premium, skill_premium $_{j t-1}$, separately for $\frac{w 90}{w 50}$ and $\frac{w 50}{w 10}$ in equations and may lead to a problem of omitted variable bias. We therefore examine in Table 14 the sensitivity of the significant effects on skill distribution of the predicted trade-induced skill premium reported in Table 3 for Denmark to unobservables, by using the method proposed by Oster (2013). This test formalizes a relationship between: i)the association between selection on observables and unobservables and ii) R-squared values. This is done in order to explain the potential importance of omitted variable bias. Movements in the coefficients and $\mathrm{R}$-squared values identify the bias resulting from omitted variables. Column (1) reports the raw effect of the predicted trade-induced skill premium from a regression of one of the two moments of the skill distribution on skill_premium prt-1 $_{j}$. Column (2) is the controlled effect, including all the observables reported in Table 3 and acts as an upper bound for the estimated effect of skill premium on one of the two moments of the skill distribution. The reported R-squared values in columns (1) and (2) correspond respectively to $\dot{R}$ and $\tilde{R}$ respectively in (Oster, 2013). Column (3) reports the bounded set taking the value from column (2) and the bound estimate assuming that: i) the relationship between the covariance, $\delta$, of the variable of interest, i.e. the skill premium, and observables and the covariance of the variable of interest and unobservables is equal to 1 , and ii) $R_{\max }$ is higher than $\tilde{R}$. When $\delta=1$, unobservables and observables are equally important. When $R_{\max }=1$, the dependent variable is fully explained by the variable of interest, and both the observable and unobservable controls. As this may not be reasonable to assume ${ }^{22}$, we set $R_{\max }=0.95$ (i.e. $R_{\max }=1.1^{*} \tilde{R}$ ) for the effects on the mean of skills and $R_{\max }=0.8$ (i.e. $R_{\max }=1.4^{*} \tilde{R}$ ) for the effects on the standard deviation of skills. The resulting bound using the procedure developed by (Oster, 2013) suggests that, using reasonable values for the necessary assumptions, we can exclude the possibility that $\frac{w 90}{w 50}$ has a negative impact on the mean and the standard deviation of skills or that $\frac{w 50}{w 10}$ has a positive effect on the mean.

Despite these relatively tight bounds, we can further investigate what properties, in terms of unobservables, would have to hold in order to explain away the whole effect of the skill premium. Column (4) reports the level of $\delta$ required for the effect of the variable of interest on mean or the standard deviation of skills to be zero. The results show that selection on unobservables would have to be greater than selection on observables, more than twice as important as selection on observables if we focus on the mean of skills as the outcome variable. (Oster, 2013) suggest equal selection, i.e. $\delta=1$, as a reasonable bound. Columns

\footnotetext{
${ }^{22}$ For example if $R_{\max }=1$, the first two moments of the skill distribution can be completely explained, implying zero measurement errors in the data.
} 
(5) and (6) present two further checks. The bound estimate of the effect on the skills mean (standard deviation) obtained by increasing $\delta$ from column (3) from 1 to 1.5 (1.3) and the bound estimate obtained by increasing the $R_{\max }$ from column (3) to 1 . Both these additional results show that the effect of skill premium changes are bounded away from zero.

We finally check whether our results are sensitive to the specification of the first-year lag in the second stage regressions. We therefore run these regressions by including either a two-year or a three-year lag instead of the one-year lag to allow more time for the skill distribution to adjust to skill premium changes induced by trade. The findings from this last check are reported in Table 13. The evidence provided by using a two-years lag is almost identical to the one obtained in the main analysis. The specification with three-years lag delivers coefficients of lower magnitudes and larger standard errors for Denmark, which is at odd with the expectation that it may take some time for the skill distribution to adjust to skill premium changes induced by trade. This may be due to some extent to a considerable reduction in sample sizes when later than two-years lags are included in the specifications. As in the baseline model, the results for Portugal are never statistically significant.

\section{Conclusion}

Our paper shows that changes in the skill premium induced by trade significantly affect the aggregate skill levels as well as variation in skills in a context where matching frictions are low and the costs of skill upgrading are rather limited, like in the Danish case. At the opposite end of this extreme case we conclude that the skill distribution doesn't seem to adjust as much to variations in the trade-induced skill premium, given that for Portugal none of the effects on the first two moments of the skill distributions are precisely estimated.

We present a simple theoretical intuition on the link between skill premium changes and skill upgrading decisions at the individual level. We further show that when individuals respond to changes in the skill premium, there can potentially be significant distributional effects at the macro level. This is because changes in skill premium affects the opportunity cost and the returns to skill upgrading, which translate into significant effects on the skill distribution for economies characterized by flexible markets and less so for inflexible markets.

Empirically, we apply a two-step empirical analysis to administrative data for both Denmark and Portugal. The first step predicts the skill premium induced by trade shocks whereas the second step estimates the effects of such predicted skill premium on the subsequent distribution of skills at the industry level. The main results for Denmark show that the effects of the skill premium changes driven by trade shocks are non-linear: increases in low-medium 
skill premium reduces mean and variance of the skills whereas the opposite is observed with increases in medium-high skill premium. Given that trade seems to affect the low-medium skill premium negatively and the medium-high skill premium positively, we can conclude that the overall distribution of skills in Denmark has to tendency to shift to the right and to become more dispersed as a result of trade. For Portugal, none of the effects on the skill distribution are statistically significant. Despite the impact on the export volumes on the wage gaps for both high and low skilled workers is of larger size for Portugal compared to Denmark, skill premium changes triggered by trade shocks don't seem to affect the overall distribution of skills in Portugal. All in all, these results are in line with our theoretical predictions as well as robust to various sensitivity tests.

This study informs the policy makers about the distributional effects of trade integration on skills which are shown to be economically and statistically significant if the framework conditions and the labor market institutions are conducive to distributional adjustments. While designing policies for labor adjustment process to trade shocks, policy makers must account for such effects, especially in a context that favors the skill upgrading of the workforce and the labor reallocation across firms within industries or across industries, like in the Danish one.

In particular, if the policy maker's ex-post trade integration objective is to reduce the overall variation in the skills within the country then the first order analysis is to assess how trade integration affects the skill premium across the different skills. If the skill premium for the high skilled workers increases, the overall variation in the skills will be magnified. Therefore, the policy design should focus on supporting the middle skills to reduce the variation in the skills without affecting the average skills. If skill premium for the lower skilled workers increases, the variation of skills is reduced but the average skill reduces as well. Therefore, a policy that uniformly assists the lower skilled workers to upgrade skills would improve the average skill without affecting the variation in skills. 


\section{References}

Abowd, J. M., Kramarz, F., and Margolis, D. N. (1999). High wage workers and high wage firms. Econometrica, 67(2):251-334.

Addison, J. T., Portugal, P., and Vilares, H. (2016). Unions and collective bargaining in the wake of the great recession. IZA Discussion Paper No. 8943.

Andersen, T. M. (2012). A flexicurity labour market in the great recession: The case of denmark. De Economist, 160(2):117-140.

Atkin, D. (2012). Endogenous skill acquisition and export manufacturing in mexico. Technical report, National Bureau of Economic Research.

Autor, D., Dorn, D., and Hanson, G. H. (2013). The China Syndrome: Local Labor Market Effects of Import Competition in the United States. IZA Discussion Papers, (7150).

Blanchard, E. and Olney, W. (2015). Globalization and human capital investment: How export composition drives educational attainment. mimeo.

Blanchard, E. J. and Willmann, G. (2016). Trade, education, and the shrinking middle class. Journal of International Economics, 99(1):263-278.

Blanchard, O. and Portugal, P. (2001). What hides behind an unemployment rate: Comparing portuguese and u.s. labor markets. The American Economic Review, 91(1):187-207.

Bombardini, M., Gallipoli, G., and Pupato, G. (2012). Skill dispersion and trade flows. American Economic Review, 102(5):2327-48.

Botero, J. C., Djankov, S., Porta, R. L., Lopez-de Silanes, F., and Shleifer, A. (2004). The regulation of labor. The Quarterly Journal of Economics, 119(4):1339-1382.

Buhai, I. S., Portela, M. A., Teulings, C. N., and van Vuuren, A. (2014). Returns to tenure or seniority? Econometrica, 82(2):705-730.

Card, D., Cardoso, A. R., and Kline, P. (2016). Bargaining and the gender wage gap: A direct assessment. The Quarterly Journal of Economics.

Card, D., Heining, J., and Kline, P. (2013). Workplace heterogeneity and the rise of west german wage inequality. The Quarterly Journal of Economics, 128(3):967-1015.

Cardoso, A. R. and Portugal, P. (2005). Contractual wages and the wage cushion under different bargaining settings. Journal of Labor Economics, 23(4):875-902. 
Colantone, I., Crino, R., and Ogliari, L. (2015). The hidden cost of globalization: Import competition and mental distress. CESifo Working Paper Series 5586, CESifo Group Munich.

Costinot, A. and Vogel, J. (2010). Matching and inequality in the world economy. Journal of Political Economy, 118(4):747-786.

Edmonds, E. V., Topalova, P., and Pavcnik, N. (2009). Child labor and schooling in a globalizing world: Some evidence from urban india. Journal of the European Economic Association, 7(2-3):498-507.

Egger, H. and Kreickemeier, U. (2009). Redistributing gains from globalisation*. The Scandinavian Journal of Economics, 111(4):765-788.

Eriksson, T. and Westergaard-Nielsen, N. (2009). Wage and labor mobility in denmark, 1980-2000. The Structure of Wages: An International Comparison, pages 101-123.

Findlay, R. and Kierzkowski, H. (1983). International trade and human capital: a simple general equilibrium model. The Journal of Political Economy, pages 957-978.

Goldberg, P. K. and Pavcnik, N. (2007). Distributional effects of globalization in developing countries. Journal of Economic Literature, 45:39-82.

Hickman, D. C. and Olney, W. W. (2011). Globalization and investment in human capital. Industrial \& Labor Relations Review, 64(4):654-672.

Hummels, D., Jorgensen, R., Munch, J., and Xiang, C. (2014). The wage effects of offshoring: Evidence from danish matched worker-firm data. American Economic Review, 104(6):1597-1629.

ILO (2014). Tackling the job crisis in portugal. ILO reports.

Iranzo, S., Schivardi, F., and Tosetti, E. (2008). Skill dispersion and firm productivity: An analysis with employer-employee matched data. Journal of Labor Economics, 26(2):247285.

Irarrazabal, A., Moxnes, A., and Ulltveit-Moe, K. H. (2013). Heterogeneous firms or heterogeneous workers? implications for exporter premiums and the gains from trade. The Review of Economics and Statistics, 95(3):839-849.

Johnson, G. and Stafford, F. (1999). The labor market implications of international trade. Handbook of Labor Economics, 3:2215-2288. 
Maggi, G. and Grossman, G. M. (2000). Diversity and trade. American Economic Review, 90(5):1255-1275.

OECD (2013). Oecd economic surveys: Denmark 2013. OECD surveys.

OECD (2014). Oecd economic surveys: Portugal 2014. OECD surveys.

Oster, E. (2013). Unobservable Selection and Coefficient Stability: Theory and Validation. NBER Working Papers 19054, National Bureau of Economic Research, Inc.

Portela, M. (2001). Measuring skill: a multi-dimensional index. Economics Letters, 72(1):2732.

Yeaple, S. R. (2005). A simple model of firm heterogeneity, international trade, and wages. Journal of international Economics, 65(1):1-20. 


\section{A Appendix: Proposition Proofs}

\section{A.1 Theoretical Solution}

In this section, we lay out a general model of skill upgrading decision. $\theta=1$ correspond to a friction less environment while $0<\theta<1$ corresponds to the frictional environment.

Since individuals can perfectly smooth their consumption over time, it is easy to show that their life-time utility $V_{i}$ is an increasing function of their life-time income $W_{i} \equiv \sum_{j=1}^{3} w\left(s_{i j}\right) p_{j}\left(1-t_{i j}\right)$. Hence, we examine the following three cases.

Case 1: No skill upgrading: $I_{i j}=0$ for $j=1,2,3$

The first case is if the individual decides not to obtain additional skills at all. One's life-time income is:

$$
W^{1}=w(\underline{s})\left(p_{1}+p_{2}+p_{3}\right)
$$

Case 2: Upgrade skill once: $I_{i 1}=1$ and $I_{i j}=0$ for $j=2,3$

The second case is if the individual upgrades skill once in the life time. One's life-time income is:

$$
W^{2}=w(\underline{s}) p_{1}\left(1-\frac{\bar{e}}{a_{i}}\right)+[\theta w(\underline{s}+\bar{e})+(1-\theta) w(\underline{s})] p_{2}+[\theta w(\underline{s}+\bar{e})+(1-\theta) w(\underline{s})] p_{3}
$$

Case 3: Upgrade skill twice: $I_{i j}=1$ for $j=1,2$ and $I_{i 3}=0$

The third case is if the individual upgrade skills twice in the life time. One's life-time income is:

$W^{3}=w(\underline{s}) p_{1}\left(1-\frac{\bar{e}}{a_{i}}\right)+[\theta w(\underline{s}+\bar{e})+(1-\theta) w(\underline{s})] p_{2}\left(1-\frac{\bar{e}}{a_{i}}\right)+[\theta w(\underline{s}+2 \bar{e})+(1-\theta) w(\underline{s}+\bar{e})] p_{3}(10$

We solve for the ability thresholds $A_{1}=\frac{w(\underline{s}) p_{1} \bar{e}}{\theta\left(p_{2}+p_{3}\right) \Delta_{1}}$ and $A_{2}=\frac{\left[w(\underline{s})+\theta \Delta_{1}\right] p_{2} \bar{e}}{p_{3}\left[\theta \Delta_{2}+(1-\theta) \Delta_{1}\right]}$ such that when an individual $i$ 's ability $a_{i} \leq A_{1}$, they have $W^{1} \geq W^{2}$ and when an individual $i^{\prime} s a_{i} \geq A_{2}$, the individual has $W^{3} \geq W^{2}$; when an individual $i$ 's ability $A_{1} \leq a_{i} \leq A_{2}$, the individual has $W^{2} \geq W^{3}$ and $W^{2} \geq W^{1}$.

The effect of changes in $\Delta_{1}$ and $\Delta_{2}$ on thresholds can be summarized as follows:

$$
\begin{aligned}
\frac{\partial A_{1}}{\partial \Delta_{1}} & =-\frac{w(\underline{s}) p_{1} \bar{e}}{\theta\left(p_{2}+p_{3}\right) \Delta_{1}^{2}}<0 \\
\left.\frac{\partial A_{2}}{\partial \Delta_{1}}\right|_{\Delta_{2}} & =\frac{p_{2} \bar{e}\left[\theta^{2} \Delta_{2}-(1-\theta) w(\underline{s})\right]}{p_{3}\left[\theta \Delta_{2}+(1-\theta) \Delta_{1}\right]^{2}} \\
\left.\frac{\partial A_{2}}{\partial \Delta_{2}}\right|_{\Delta_{1}} & =-\frac{\theta\left[w(\underline{s})+\theta \Delta_{1}\right] p_{2} \bar{e}}{p_{3}\left[\theta \Delta_{2}+(1-\theta) \Delta_{1}\right]^{2}}<0
\end{aligned}
$$


Moreover, changes in $\theta$ affecting the above differentials can be summarized as follows:

$$
\begin{aligned}
\frac{\partial\left(\frac{\partial A_{1}}{\partial \Delta_{1}}\right)}{\partial \theta} & =\frac{w(\underline{s}) p_{1} \bar{e}}{\theta^{2}\left(p_{2}+p_{3}\right) \Delta_{1}^{2}}>0 \\
\frac{\partial\left(\left.\frac{\partial A_{2}}{\partial \Delta_{1}}\right|_{\Delta_{2}}\right)}{\partial \theta} & =\frac{p_{2} \bar{e}\left[w(\underline{s})\left(2 \Delta_{2}-\Delta_{1}\right)+\theta w(\underline{s})\left(\Delta_{1}-\Delta_{2}\right)\right]}{p_{3}\left[\theta \Delta_{2}+(1-\theta) \Delta_{1}\right]^{3}} \\
\frac{\partial\left(\left.\frac{\partial A_{2}}{\partial \Delta_{2}}\right|_{\Delta_{1}}\right)}{\partial \theta} & =-\frac{p_{2} \bar{e}\left[w(\underline{s}) \Delta_{1}+w(\underline{s}) \theta\left(\Delta_{1}-\Delta_{2}\right)+2 \theta \Delta_{1}^{2}\right]}{p_{3}\left[\theta \Delta_{2}+(1-\theta) \Delta_{1}\right]^{3}}<0
\end{aligned}
$$

Last, assuming uniform distribution of ability, the first two moments are computed using the following expressions. Moreover, the movement of threshold in response to changes in $\Delta$ 's affect these moments and we provide the corresponding implications below:

(1) Mean $(E(s))$ :

$$
\begin{aligned}
E(s) & =\underline{s} \frac{A_{1}-\underline{a}}{\bar{a}-\underline{a}}+(\underline{s}+\bar{e}) \frac{A_{2}-A_{1}}{\bar{a}-\underline{a}}+(\underline{s}+2 \bar{e}) \frac{\bar{a}-A_{2}}{\bar{a}-\underline{a}} \\
& =\underline{s}+\frac{2 \bar{a}-A_{1}-A_{2}}{\bar{a}-\underline{a}} \bar{e}
\end{aligned}
$$

(2) Variance $(\operatorname{Var}(s))$ :

$$
\begin{aligned}
\operatorname{Var}(s) & =\sum\left[s_{i}-E(s)\right]^{2} \\
& =\frac{A_{1}-\underline{a}}{\bar{a}-\underline{a}}\left(\frac{2 \bar{a}-A_{1}-A_{2}}{\bar{a}-\underline{a}} \bar{e}\right)^{2}+\frac{A_{2}-A_{1}}{\bar{a}-\underline{a}}\left(\frac{\bar{a}+\underline{a}-A_{1}-A_{2}}{\bar{a}-\underline{a}}\right)^{2}+\frac{\bar{a}-A_{2}}{\bar{a}-\underline{a}}\left(\frac{2 \underline{a}-A_{1}-A_{2}}{\bar{a}-\underline{a}}\right)^{2} \\
& =\frac{\bar{e}^{2}}{(\bar{a}-\underline{a})^{3}}\left\{\left(A_{1}-\underline{a}\right)\left(2 \bar{a}-A_{1}-A_{2}\right)^{2}+\left(A_{2}-A_{1}\right)\left(\bar{a}+\underline{a}-A_{1}-A_{2}\right)^{2}\right. \\
& \left.+\left(\bar{a}-A_{2}\right)\left(2 \underline{a}-A_{1}-A_{2}\right)^{2}\right\} \\
& =\frac{\bar{e}^{2}}{(\bar{a}-\underline{a})^{3}}\left\{\left(A_{1}-\underline{a}\right)\left(2 \bar{a}-A_{1}-A_{2}\right)^{2}+\left[\bar{a}-\underline{a}-\left(A_{1}-\underline{a}\right)-\left(\bar{a}-A_{2}\right)\right]\left(\bar{a}+\underline{a}-A_{1}-A_{2}\right)^{2}\right. \\
& \left.+\left(\bar{a}-A_{2}\right)\left(2 \underline{a}-A_{1}-A_{2}\right)^{2}\right\} \\
& =\frac{\bar{e}^{2}}{(\bar{a}-\underline{a})^{3}}\left\{\left(A_{1}-\underline{a}\right)\left[\left(2 \bar{a}-A_{1}-A_{2}\right)^{2}-\left(\bar{a}+\underline{a}-A_{1}-A_{2}\right)^{2}\right]+[\bar{a}-\underline{a}]\left(\bar{a}+\underline{a}-A_{1}-A_{2}\right)^{2}\right. \\
& \left.+\left(\bar{a}-A_{2}\right)\left[\left(2 \underline{a}-A_{1}-A_{2}\right)^{2}-\left(\bar{a}+\underline{a}-A_{1}-A_{2}\right)^{2}\right]\right\} \\
& =\frac{\bar{e}^{2}}{(\bar{a}-\underline{a})^{2}}\left\{\left(A_{1}-\underline{a}\right)\left(3 \bar{a}+\underline{a}-2 A_{1}-2 A_{2}\right)+\left(\bar{a}-A_{2}\right)\left(2 A_{1}+2 A_{2}-\bar{a}-3 \underline{a}\right)\right. \\
& \left.+\left(\bar{a}+\underline{a}-A_{1}-A_{2}\right)^{2}\right\} \\
& =\frac{\bar{e}^{2}}{(\bar{a}-\underline{a})^{2}}\left\{\left(A_{1}-\underline{a}\right)\left(3 \bar{a}+\underline{a}-2 A_{1}-2 A_{2}\right)-\bar{a} \underline{a}+\left(A_{1}-\underline{a}\right)^{2}+A_{2}\left(\bar{a}+\underline{a}-A_{2}\right)\right\}
\end{aligned}
$$




\section{A.2 Flexible Market $(\theta=1)$}

Replacing $\theta=1, a_{i} \leq \frac{w(\underline{s}) p_{1} \bar{e}}{\left(p_{2}+p_{3}\right)[w(\underline{s}+\bar{e})-w(\underline{s})]} \equiv A_{1}$ and $a_{i} \geq \frac{w(\underline{s}+\bar{e}) p_{2} \bar{e}}{p_{3}[w(\underline{s}+2 \bar{e})-w(\underline{s}+\bar{e})]} \equiv A_{2}$. Assuming $\frac{d^{2} w}{d s^{2}}<0$ and positive inflation (i.e., $p_{1} \leq p_{2} \leq p_{3}$ ), we have $A_{1}<A_{2}$. Hence, we can draw Figure 1 .

Figure 1: Ability Thresholds and Skill Upgrading Decision

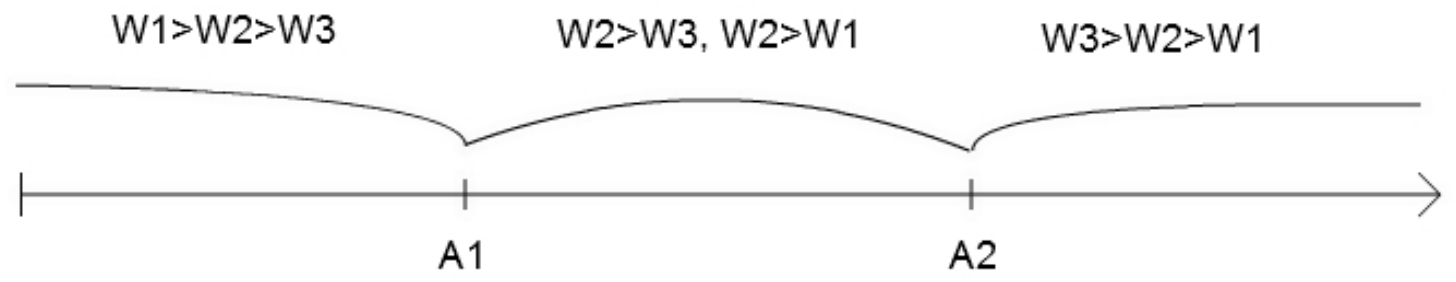

Note: The above illustration presents the ability specific thresholds and the associated ranking of wealth with zero, once or twice skill upgrading. This illustration highlights that those individuals whose ability is below $A_{1}$ do not upgrade skills at all, those with above $A_{2}$ level of ability upgrade skills twice in their life time, and those in between $A_{1}$ and $A_{2}$ upgrade skills only once.

$$
\begin{aligned}
\frac{\partial A_{1}}{\partial \Delta_{1}} & <0 \\
\left.\frac{\partial A_{2}}{\partial \Delta_{1}}\right|_{\Delta_{2}} & >0 \\
\left.\frac{\partial A_{2}}{\partial \Delta_{2}}\right|_{\Delta_{1}} & <0
\end{aligned}
$$

\section{Proof of Proposition 1.}

- If $\left|\frac{\partial A_{1}}{\partial \Delta_{1}}\right|<\left|\frac{\partial A_{2}}{\partial \Delta_{1}}\right|_{\Delta_{2}} \mid$, an increase in $\Delta_{1}$ decreases mean $(E(s))$ and variation $(\operatorname{Var}(s))$ of skills. (1) Mean: When $\Delta_{1}$ increases, $A_{1}$ decreases and $A_{2}$ increases. Since $\left|\frac{\partial A_{1}}{\partial \Delta_{1}}\right|<\left|\frac{\partial A_{2}}{\partial \Delta_{1}}\right|_{\Delta_{2}} \mid$, the decrease of $A_{1}$ is smaller than the increase of $A_{2}$. Hence, $E(s)=\underline{s}+\frac{2 \bar{a}-A_{1}-A_{2}}{\bar{a}-\underline{e}} \bar{e}$ decreases. (2) Variance: Since $\bar{a}+\underline{a}<2 A_{2}, \bar{a}+\underline{a}>2 A_{1}$, and $\underline{a}<A_{1}<A_{2}<\bar{a}$, we have $3 \bar{a}+\underline{a}-$ $2 A_{1}-2 A_{2}>\bar{a}+\underline{a}-2 A_{1}>0$. When $\Delta_{1}$ increases, $A_{1}$ decreases and $A_{2}$ increases. Assuming $\left|\frac{\partial A_{1}}{\partial \Delta_{1}}\right|<\left|\frac{\partial A_{2}}{\partial \Delta_{1}}\right|_{\Delta_{2}} \mid$, we have $\operatorname{both}\left(A_{1}-\underline{a}\right)$ and $\left(3 \bar{a}+\underline{a}-2 A_{1}-2 A_{2}\right)$ decrease. The last term in $\operatorname{Var}(s)$ is $A_{2}\left(\bar{a}+\underline{a}-A_{2}\right)$. Its value changes by $\bar{a}+\underline{a}-2 A_{2}<0$ when $A_{2}$ increases by 1. Hence, $\operatorname{Var}(s)$ is $A_{2}\left(\bar{a}+\underline{a}-A_{2}\right)$ also decreases when $A_{2}$ increases. Therefore, we prove $\operatorname{Var}(s)$ decreases when $\Delta_{1}$ increases.

- An increase in $\Delta_{2}$ increases both the mean $(E(s))$ and the variation $(\operatorname{Var}(s))$ of skills. (1) Mean: When $\Delta_{2}$ increases, $A_{2}$ decreases while $A_{1}$ does not change. Hence, $E(s)=$ $\underline{s}+\frac{2 \bar{a}-A_{1}-A_{2}}{\bar{a}-\underline{a}} \bar{e}$ increases.

(2) Variance: When $A_{2}$ decreases, we have both $3 \bar{a}+\underline{a}-2 A_{1}-2 A_{2}$ and $A_{2}\left(\bar{a}+\underline{a}-A_{2}\right)$ increases. Therefore, we prove $\operatorname{Var}(s)$ increases when $\Delta_{2}$ increases. 


\section{A.3 Frictional Markets $(\theta \neq 1)$}

Before proving Proposition 2, we first establish the condition for $\theta$ under which $A_{1}<A_{2}$. In order to have $A_{1}<A_{2}$, we need $X \theta^{2}+Y \theta+Z>0$ where

$$
\begin{aligned}
X & =\left(p_{2}+p_{3}\right) p_{2} \bar{e} \Delta_{1}^{2}>0 \\
Y & =\bar{e} w(\underline{s})\left[\left(p_{2}+p_{3}\right) p_{2} \Delta_{1}+\left(\Delta_{1}-\Delta_{2}\right) p_{1} p_{3}\right]>0 \\
Z & =-\bar{e} w(\underline{s}) p_{1} p_{3} \Delta_{1}<0
\end{aligned}
$$

Given the quadratic nature of the equation $X \theta^{2}+Y \theta+Z$, it is easy to see that the equation's lowest point is achieved at $\theta=\frac{-Y}{2 X}<0$. That is, for any $0<\theta \leq 1, X \theta^{2}+Y \theta+Z$ monotonically increases along $\theta$. Then we solve for the positive $\theta^{*}$ that equalizes $X \theta^{2}+Y \theta+Z$ to 0 :

$$
\theta^{*}=\frac{-Y+\sqrt{Y^{2}-4 X Z}}{2 X}>0
$$

where $-4 X Z>0$ and thus $Y^{2}-4 X Z>Y^{2}>0$. Hence, we have $\frac{-Y+\sqrt{Y^{2}-4 X Z}}{2 X}>0$. Assuming $\frac{d^{2} w}{d s^{2}}<0$ and positive inflation (i.e., $p_{1} \leq p_{2} \leq p_{3}$ ), when $\theta=1$ in the baseline model we have $A_{1}<A_{2}$ and thus $X \theta^{2}+Y \theta+Z>0$. Therefore, given $X \theta^{2}+Y \theta+Z$ monotonically increasing for positive $\theta \in\left[\theta^{*}, 1\right]$.

We have proven that $\frac{-Y+\sqrt{Y^{2}-4 X Z}}{2 X}<1$. Hence, we've shown that in order to have $A_{1}<A_{2}$, we need $0<\frac{-Y+\sqrt{Y^{2}-4 X Z}}{2 X}=\theta^{*}<\theta \leq 1$, and this condition is consistent with $\theta$ being a probability between 0 and 1 .

Restricting $\theta \in\left[\theta^{*}, 1\right]$, we can now study the distributional effects of changes in skill premium.

Recall that we cannot sign the $\left.\frac{\partial A_{2}}{\partial \Delta_{1}}\right|_{\Delta_{2}}$ unless we have additional conditions stating whether $\theta$ is above or below some cut-off denoted by $\bar{\epsilon}$ while restricting the cut-off to lie in the range where $A_{1}<A_{2}$ only i.e., $\theta^{*}<\bar{\epsilon}$. To find $\theta=\bar{\epsilon}$ we need the numerator of $\left.\frac{\partial A_{2}}{\partial \Delta_{1}}\right|_{\Delta_{2}}=0$ which implies that $\left[\theta^{2} \Delta_{2}-(1-\theta) w(\underline{s})\right]=0$. Solving for the quadratic equation in $\theta, \bar{\epsilon}=\frac{-w(\underline{s}) \pm \sqrt{w^{2}(\underline{s})+4 \Delta_{2} w(\underline{s})}}{2 \Delta_{2}}$.

Then for $\theta \in\left[\theta^{*}, \bar{\epsilon}\right]$ we have $\left.\frac{\partial A_{2}}{\partial \Delta_{1}}\right|_{\Delta_{2}}<0$ and for $\theta \in[\bar{\epsilon}, 1]$ we have $\left.\frac{\partial A_{2}}{\partial \Delta_{1}}\right|_{\Delta_{2}}>0$.

\section{Proof of Proposition 2.}

- An increase in $\Delta_{1}$ has ambiguous effect on $E(s)$ and $\operatorname{Var}(s)$. Depending on the relation between $\left|\frac{\partial A_{1}}{\partial \Delta_{1}}\right|$ and $\left|\frac{\partial A_{2}}{\partial \Delta_{1}}\right|_{\Delta_{2}} \mid$, and the magnitude of $\theta$ various cases are possible and therefore 
the ultimate effect is at best ambiguous.

$$
\begin{gathered}
\text { Mean }=\left\{\begin{array}{l}
\text { Decreases if }\left|\frac{\partial A_{1}}{\partial \Delta_{1}}\right|<\left|\frac{\partial A_{2}}{\partial \Delta_{1}}\right|_{\Delta_{2}} \mid \& \theta \in[\bar{\epsilon}, 1] \\
\text { Increases if }\left|\frac{\partial A_{1}}{\partial \Delta_{1}}\right|>\left|\frac{\partial A_{2}}{\partial \Delta_{1}}\right|_{\Delta_{2}} \mid \& \theta \in[\bar{\epsilon}, 1] \\
\text { Unchanged if }\left|\frac{\partial A_{1}}{\partial \Delta_{1}}\right|=\left|\frac{\partial A_{2}}{\partial \Delta_{1}}\right|_{\Delta_{2}} \mid \& \theta \in[\bar{\epsilon}, 1] \\
\text { Increases if } \theta \in\left[\theta^{*}, \bar{\epsilon}\right]
\end{array}\right. \\
\text { Variance }=\left\{\begin{array}{l}
\text { Decreases if }\left|\frac{\partial A_{1}}{\partial \Delta_{1}}\right|<\left|\frac{\partial A_{2}}{\partial \Delta_{1}}\right|_{\Delta_{2}} \mid \& \theta \in[\bar{\epsilon}, 1] \\
\text { Ambiguous otherwise }
\end{array}\right.
\end{gathered}
$$

- An increase in $\Delta_{2}$ increases both $E(s)$ and $\operatorname{Var}(s)$ of skills but by a smaller magnitude relative to when $\theta=1$. (1) Mean: When $\Delta_{2}$ increases, $A_{2}$ decreases while $A_{1}$ does not change. Hence, $E(s)=\underline{s}+\frac{2 \bar{a}-A_{1}-A_{2}}{\bar{a}-\underline{a}} \bar{e}$ increases. Moreover, $\frac{\partial\left(\left.\frac{\partial A_{2}}{\partial \Delta_{2}}\right|_{1}\right)}{\partial \theta}<0$, meaning in absolute terms the effect increases as $\theta$ increases.

(2) Variance: When $A_{2}$ decreases, we have both $3 \bar{a}+\underline{a}-2 A_{1}-2 A_{2}$ and $A_{2}\left(\bar{a}+\underline{a}-A_{2}\right)$ increases. Therefore, we prove $\operatorname{Var}(s)$ increases when $\Delta_{2}$ increases. Moreover, $\frac{\partial\left(\left.\frac{\partial A_{2}}{\partial \Delta_{2}}\right|_{\Delta_{1}}\right)}{\partial \theta}<0$, meaning in absolute terms the effect increases as $\theta$ increases. 


\section{B Appendix: Figures}

Figure 2: Danish export Volumes over GDP, Source: Statistics Denmark.

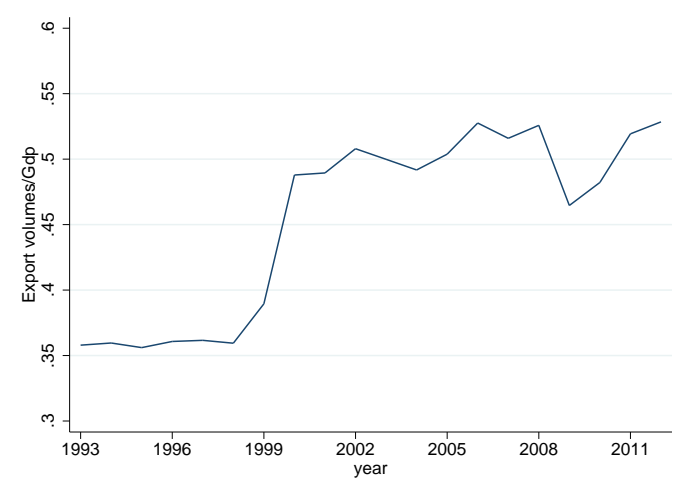

Source: Both export volumes and GDP figures are in real terms and in dkk.

Figure 3: Portuguese export Volumes over GDP, Source: Statistics Portugal.

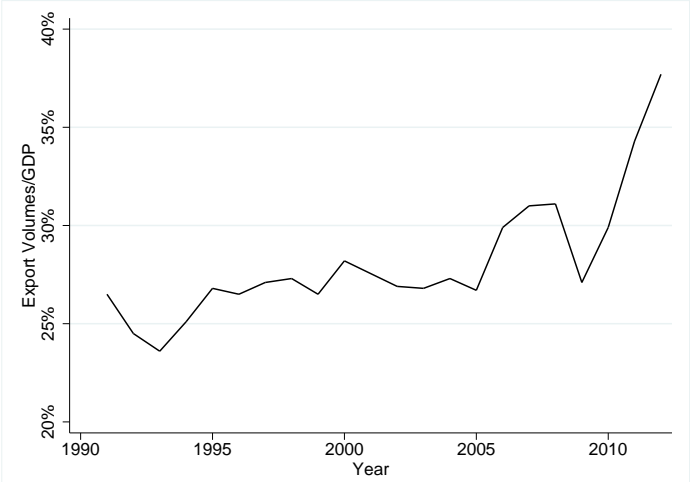

Source: Both export volumes and GDP figures are in real terms and in euros. 
Figure 4: Destination of Danish Exports, Source: Statistics Denmark.

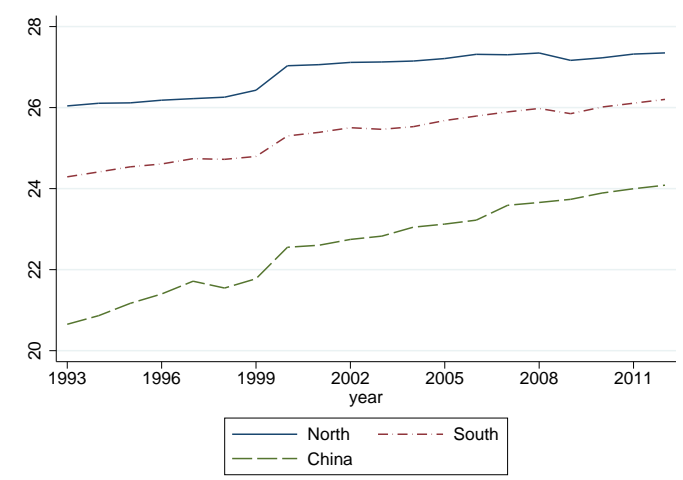

Source: Amount in dkk. North includes countries of North America, Oceania, Western and Southern Europe (excluding the new EU members of the 2004 enlargement). All the other countries in the world are considered under the category of South.

Figure 5: Destination of Portuguese Exports, Source: Statistics Portugal.

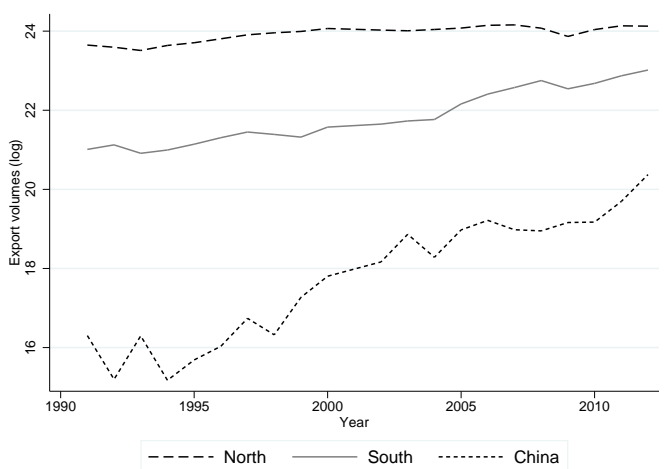

Source: Amount in euros. North includes countries of North America, Oceania, Western and Southern Europe (excluding the new EU members of the 2004 enlargement). All the other countries in the world are considered under the category of South. 
Figure 6: Overtime wage ratios and skills, Denmark
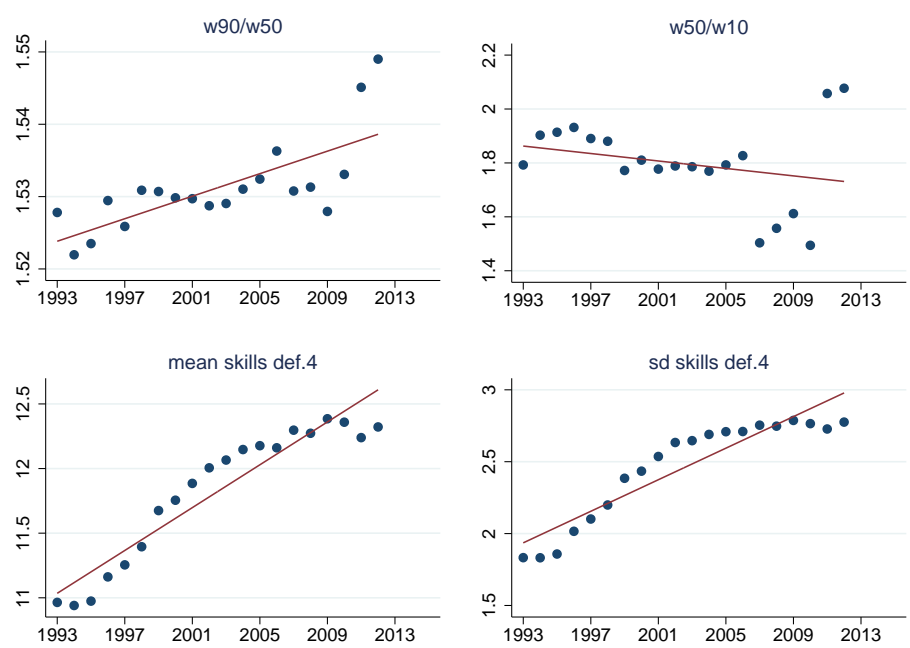

Source: The first two panels plot the average skill premium $\frac{w 90}{w 50}$ and $\frac{w 50}{w 10}$ against year. The last two panels plot the first two moments of the skill distribution against year. Skill definition 4 is based on years of education.

Figure 7: Overtime wage ratios and skills, Portugal
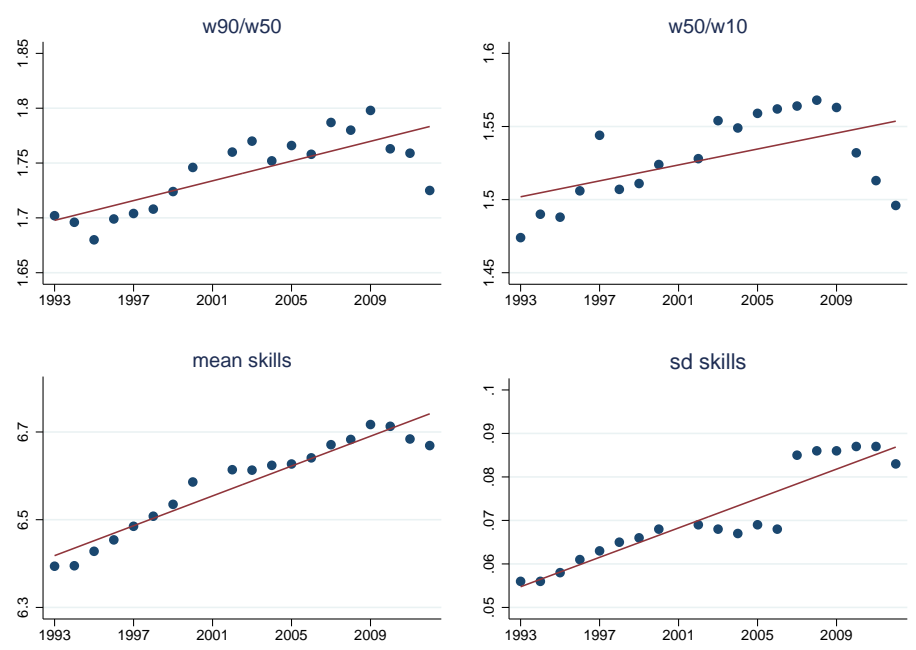

Source: The first two panels plot the average skill premium $\frac{w 90}{w 50}$ and $\frac{w 50}{w 10}$ against year. The last two panels plot the first two moments of the skill distribution against year. Skill definition 4 is based on years of education. 
Figure 8: Wage Dynamics of Male Movers.

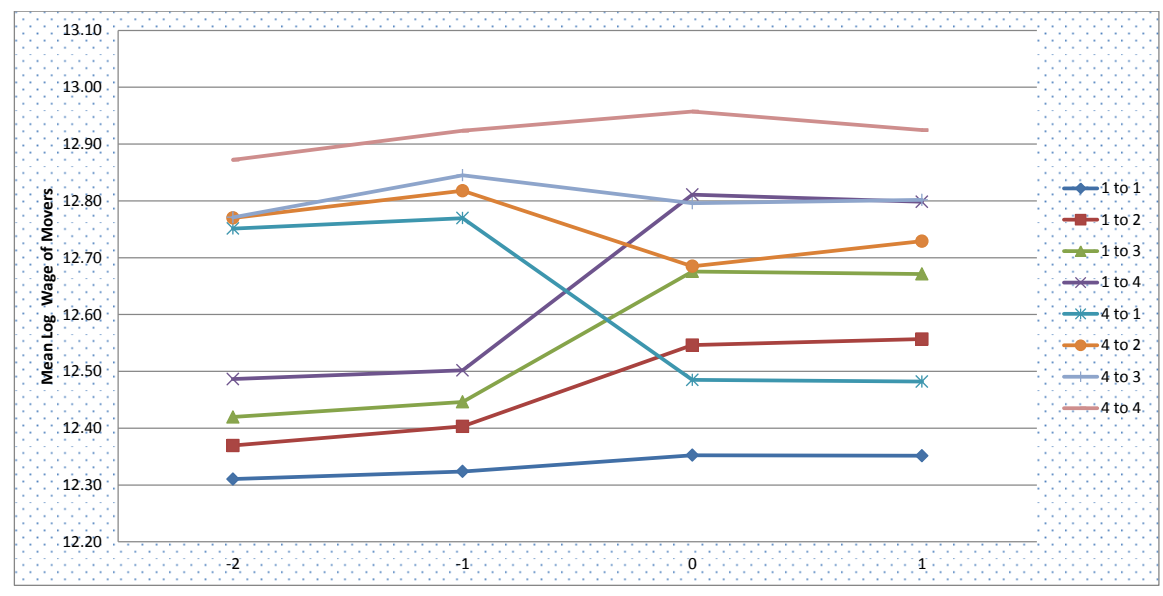

Source: Figure shows mean wages of male workers who changed jobs in 1996-2005 and held the preceding job for 2 or more years, and the new job for 2 or more years. Each job is classified into quartiles based on mean log wage of co-workers in the last year of the old job (for origin firm) and in the first year on the new job (for the destination firm).

Figure 9: Wage Dynamics of Female Movers.

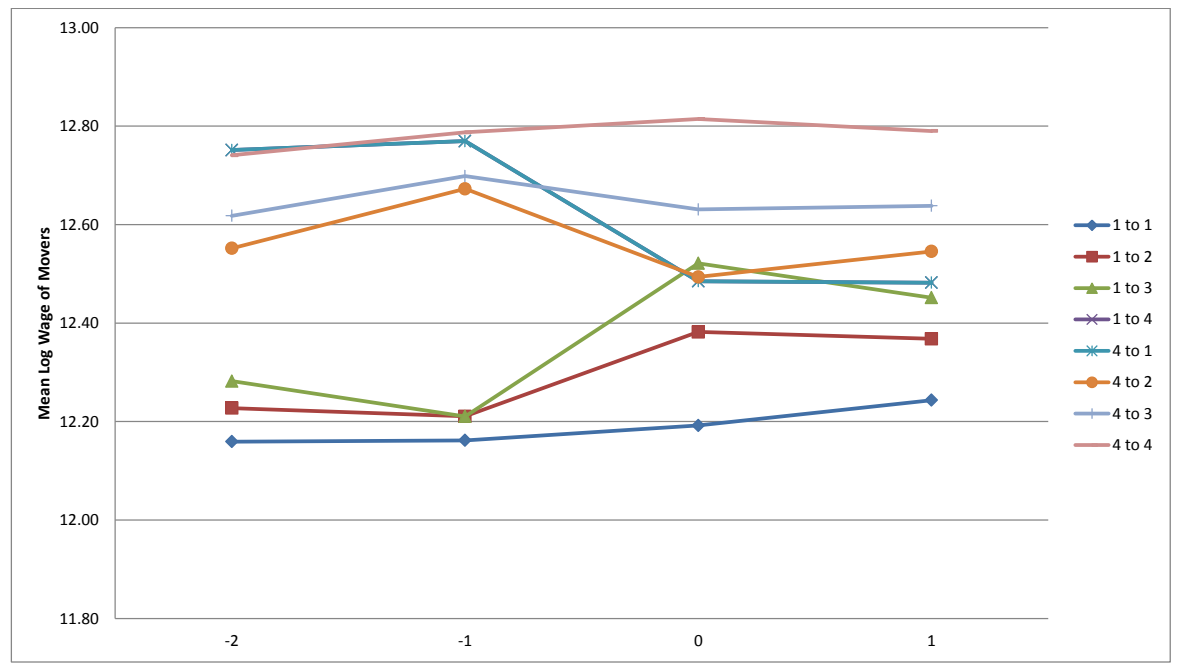

Source: Figure shows mean wages of female workers who changed jobs in 1996-2005 and held the preceding job for 2 or more years, and the new job for 2 or more years. Each job is classified into quartiles based on mean log wage of co-workers in the last year of the old job (for origin firm) and in the first year on the new job (for the destination firm). 
C Appendix: Tables 


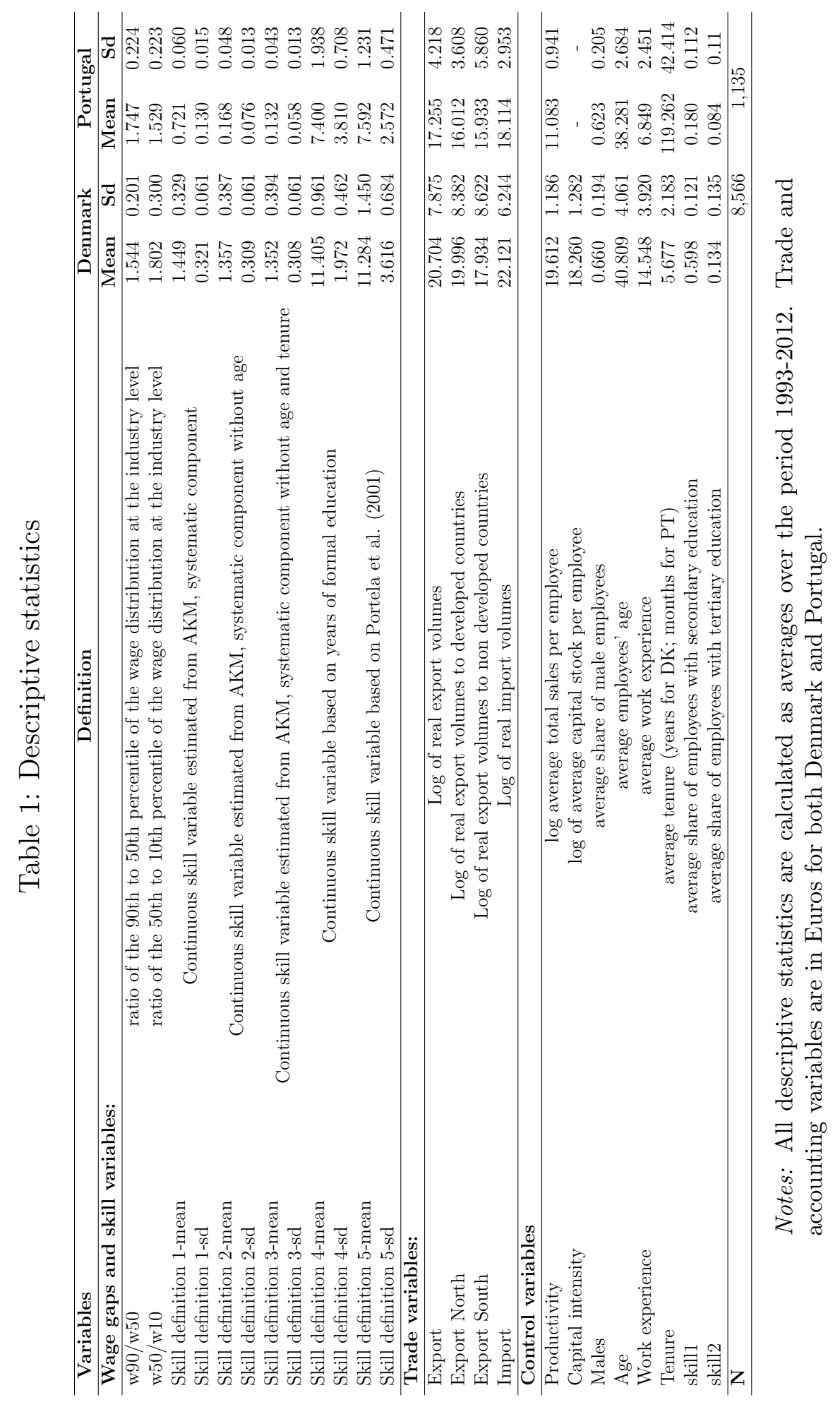


Table 2: Estimated coefficients of the time variant personal characteristics, AKM model.

\begin{tabular}{lcccc}
\hline & \multicolumn{2}{c}{ Denmark } & \multicolumn{2}{c}{ Portugal } \\
\hline age & Men & Women & Men & Women \\
age $\hat{2}$ & $0.042^{* * *}$ & $0.023^{* * *}$ & $0.033^{* * *}$ & $0.013^{* * *}$ \\
& $(0.002)$ & $(0.003)$ & $(0.000)$ & $(0.000)$ \\
work experience & $-0.001^{* * *}$ & $-0.000^{* * *}$ & $-0.000^{* * *}$ & $-0.000^{* * *}$ \\
& $(0.000)$ & $(0.000)$ & $(0.000)$ & $(0.000)$ \\
work experience2 $\hat{2}$ & $0.048^{* * *}$ & $0.062^{* * *}$ & $0.025^{* * *}$ & $0.022^{* * *}$ \\
& $(0.001)$ & $(0.003)$ & $(0.000)$ & $(0.000)$ \\
tenure & $-0.001^{* * *}$ & $-0.001^{* * *}$ & $-0.001^{* * *}$ & $-0.001^{* * *}$ \\
& $(0.000)$ & $(0.000)$ & $(0.000)$ & $(0.000)$ \\
tenure2 & $0.014^{* * *}$ & $0.014^{* * *}$ & $0.000^{* * *}$ & $0.000^{* * *}$ \\
& $(0.001)$ & $(0.002)$ & $(0.000)$ & $(0.000)$ \\
\hline Secondary education*year dummies & Yes & Yes & Yes & Yes \\
Tertiary education*year dummies & Yes & Yes & Yes & Yes \\
\hline N & $16,569,318$ & $11,238,102$ & $13,306,912$ & $10,072,610$ \\
R-sq & 0.767 & 0.743 & 0.8449 & 0.8983 \\
\hline
\end{tabular}

Notes: The dependent variable is log of annual (monthly) net earnings for Denmark (Portugal). Standard errors are clustered at the firm level. Significance levels: ${ }^{* *} 1 \%,{ }^{*} 5 \%,{ }^{*} 10 \%$. 
Table 3: Effects of Trade Activity on the Skill Premium and Distribution.

\begin{tabular}{|c|c|c|c|c|}
\hline & \multicolumn{4}{|c|}{ Denmark } \\
\hline Step 1 & w90/w50 & w50/w10 & & \\
\hline Export & $\begin{array}{c}0.002^{* * *} \\
(0.000)\end{array}$ & $\begin{array}{c}-0.005^{* * *} \\
(0.001)\end{array}$ & & \\
\hline F stat & 3074.35 & 3074.35 & & \\
\hline $\mathrm{N}$ & 8,566 & 8,566 & & \\
\hline R-sq & 0.156 & 0.407 & & \\
\hline Step 2, skill definition 1 & Mean & SD & Mean & SD \\
\hline First year lag of predicted (w90/w50) & $\begin{array}{c}0.692^{* *} \\
(0.294)\end{array}$ & $\begin{array}{c}0.178^{* *} \\
(0.088)\end{array}$ & & \\
\hline First year lag of predicted (w50/w10) & & & $\begin{array}{c}-0.318^{* *} \\
(0.159)\end{array}$ & $\begin{array}{c}-0.082 \\
(0.056)\end{array}$ \\
\hline $\mathrm{N}$ & 8,566 & 8,566 & 8,566 & 8,566 \\
\hline \multirow[t]{2}{*}{ R-sq } & 0.893 & 0.567 & 0.893 & 0.567 \\
\hline & \multicolumn{4}{|c|}{ Portugal } \\
\hline Step 1 & w90/w50 & w50/w10 & & \\
\hline Export & $\begin{array}{c}0.036^{* * *} \\
(0.004)\end{array}$ & $\begin{array}{c}0.020^{* * *} \\
(0.004)\end{array}$ & & \\
\hline F stat & 256.46 & 256.46 & & \\
\hline $\mathrm{N}$ & 1,137 & 1,137 & & \\
\hline R-sq & 0.561 & 0.595 & & \\
\hline Step 2, skill definition 1 & Mean & SD & Mean & SD \\
\hline First year lag of predicted (w90/w50) & $\begin{array}{c}-0.015 \\
(0.011)\end{array}$ & $\begin{array}{c}0.009 \\
(0.007)\end{array}$ & & \\
\hline First year lag of predicted (w50/w10) & & & $\begin{array}{l}-0.027 \\
(0.022)\end{array}$ & $\begin{array}{c}0.017 \\
(0.017)\end{array}$ \\
\hline $\mathrm{N}$ & 1,064 & 1,064 & 1,064 & 1,064 \\
\hline R-sq & 0.966 & 0.754 & 0.966 & 0.754 \\
\hline
\end{tabular}

Notes: Skill definition 1 is based on the whole systematic component of the two-way fixed effects wage regression. In step 1 , standard errors are clustered at the industry level. In step 2, standard errors are clustered at the industry level and sequentially bootstrapped with step 1, 200 replications. All estimations are also weighted for the number of firms in each industry. Both steps also include the following control variables: sales per employee, capital intensity (only for Denmark), workforce composition characteristics, year and 2 digit industry dummies. Significance levels: ${ }^{* * *} 1 \%,{ }^{* *} 5 \%$, $* 10 \%$. 
Table 4: Effects of Trade Activity on the Skill Premium and Distribution, alternative skill definitions, Denmark.

\begin{tabular}{|c|c|c|c|c|}
\hline Step 2, skill definition 2 & Mean & SD & Mean & SD \\
\hline First year lag of predicted (w90/w50) & $\begin{array}{c}0.663^{* *} \\
(0.289)\end{array}$ & $\begin{array}{c}0.181 \\
(0.131)\end{array}$ & & \\
\hline First year lag of predicted (w50/w10) & & & $\begin{array}{c}-0.305^{* *} \\
(0.151)\end{array}$ & $\begin{array}{c}-0.083 \\
(0.052)\end{array}$ \\
\hline $\mathrm{N}$ & 8,566 & 8,566 & 8,566 & 8,566 \\
\hline R-sq & 0.793 & 0.547 & 0.793 & 0.544 \\
\hline Step 2, skill definition 3 & Mean & SD & Mean & SD \\
\hline First year lag of predicted (w90/w50) & $\begin{array}{c}0.663^{* *} \\
(0.337)\end{array}$ & $\begin{array}{c}0.182 \\
(0.138)\end{array}$ & & \\
\hline First year lag of predicted (w50/w10) & & & $\begin{array}{c}-0.305^{* *} \\
(0.151)\end{array}$ & $\begin{array}{c}-0.083^{* *} \\
(0.042) \\
\end{array}$ \\
\hline $\mathrm{N}$ & 8,566 & 8,566 & 8,566 & 8,566 \\
\hline R-sq & 0.813 & 0.527 & 0.823 & 0.527 \\
\hline Step 2, skill definition 4 & Mean & SD & Mean & SD \\
\hline First year lag of predicted (w90/w50) & $\begin{array}{c}2.017^{* * *} \\
(0.752)\end{array}$ & $\begin{array}{c}2.775^{* * *} \\
(0.835)\end{array}$ & & \\
\hline First year lag of predicted (w50/w10) & & & $\begin{array}{c}-0.657^{* * *} \\
(0.193)\end{array}$ & $\begin{array}{c}-0.904^{* * * *} \\
(0.256)\end{array}$ \\
\hline $\mathrm{N}$ & 8,566 & 8,566 & 8,566 & 8,566 \\
\hline R-sq & 0.870 & 0.597 & 0.870 & 0.597 \\
\hline Step 2, skill definition 5 & Mean & SD & Mean & SD \\
\hline First year lag of predicted (w90/w50) & $\begin{array}{l}1.769^{*} \\
(1.004)\end{array}$ & $\begin{array}{c}3.152^{* * *} \\
(1.068)\end{array}$ & & \\
\hline First year lag of predicted (w50/w10) & & & $\begin{array}{c}-0.576 \\
(0.416)\end{array}$ & $\begin{array}{c}-1.026^{* * *} \\
(0.317)\end{array}$ \\
\hline $\mathrm{N}$ & 8,566 & 8,566 & 8,566 & 8,566 \\
\hline R-sq & 0.703 & 0.527 & 0.703 & 0.527 \\
\hline
\end{tabular}

Notes: Skill definitions 2 and 3 are based on the systematic component of the two-way fixed effects wage regression respectively without age and without age and tenure. Skill definition 4 is based on years of education whereas skill definition 5 is the multidimensional index as in Portela et al. (2001). Both steps also include the following control variables: sales per employee, capital intensity, workforce composition characteristics, year and 2 digit industry dummies. In step 2, standard errors are clustered at the industry level and sequentially bootstrapped with step 1, 200 replications. All estimations are also weighted for the number of firms in each industry. Significance levels: $* * * 1 \%$, ${ }^{*} * 5 \%, * 10 \%$. 
Table 5: Effects of Trade Activity on the Skill Premium and Distribution, alternative skill definitions, Portugal.

\begin{tabular}{lcccc}
\hline Step 2, skill definition 2 & Mean & SD & Mean & SD \\
\hline First year lag of predicted (w90/w50) & -0.005 & -0.001 & & \\
& $(0.009)$ & $(0.006)$ & & \\
First year lag of predicted (w50/w10) & & & -0.010 & -0.002 \\
& & & $(0.019)$ & $(0.012)$ \\
\hline $\mathrm{N}$ & 1,064 & 1,064 & 1,064 & 1,064 \\
$\mathrm{R}-\mathrm{sq}$ & 0.957 & 0.857 & 0.957 & 0.857 \\
\hline Step 2, skill definition 3 & Mean & $\mathrm{SD}$ & Mean & $\mathrm{SD}$ \\
\hline First year lag of predicted (w90/w50) & -0.003 & -0.001 & & \\
& $(0.008)$ & $(0.005)$ & & \\
First year lag of predicted (w50/w10) & & & -0.006 & -0.001 \\
& & & $(0.016)$ & $(0.008)$ \\
\hline $\mathrm{N}$ & 1,064 & 1,064 & 1,064 & 1,064 \\
$\mathrm{R}-\mathrm{sq}$ & 0.954 & 0.923 & 0.954 & 0.923 \\
\hline Step 2, skill definition 4 & Mean & $\mathrm{SD}$ & $\mathrm{Mean}$ & $\mathrm{SD}$ \\
\hline First year lag of predicted (w90/w50) & -0.501 & -0.246 & & \\
& $(0.430)$ & $(0.158)$ & & \\
First year lag of predicted (w50/w10) & & & -0.899 & -0.442 \\
& & & $(0.749)$ & $(0.342)$ \\
\hline $\mathrm{N}$ & 1,064 & 1,064 & 1,064 & 1,064 \\
$\mathrm{R}-\mathrm{sq}$ & 0.971 & 0.783 & 0.971 & 0.783 \\
\hline Step 2, skill definition 5 & Mean & $\mathrm{SD}$ & Mean & $\mathrm{SD}$ \\
\hline First year lag of predicted (w90/w50) & -0.426 & -0.003 & & \\
& $(0.279)$ & $(0.132)$ & & \\
First year lag of predicted (w50/w10) & & & -0.766 & -0.005 \\
& & & $(0.591)$ & $(0.230)$ \\
\hline $\mathrm{N}$ & 1,064 & 1,064 & 1,064 & 1,064 \\
R-sq & 0.932 & 0.914 & 0.932 & 0.914 \\
\hline
\end{tabular}

Notes: Skill definitions 2 and 3 are based on the systematic component of the two-way fixed effects wage regression respectively without age and without age and tenure. Skill definition 4 is based on years of education whereas skill definition 5 is the multidimensional index as in Portela et al. (2001). Both steps also include the following control variables: sales per employee, workforce composition characteristics, year and 2 digit industry dummies. In step 2, standard errors are clustered at the industry level and sequentially bootstrapped with step 1, 200 replications. All estimations are also weighted for the number of firms in each industry. Significance levels: ${ }^{* * *} 1 \%,{ }^{*} 5 \%,{ }^{*} 10 \%$. 
Table 6: Effects of Trade Activity on the Skill Premium and Distribution, the role of export destinations.

\begin{tabular}{|c|c|c|c|c|}
\hline & \multicolumn{4}{|c|}{ Denmark } \\
\hline Step 1 & w90/w50 & w50/w10 & & \\
\hline \multirow[t]{2}{*}{ Export North } & 0.001 & -0.001 & & \\
\hline & $(0.001)$ & $(0.002)$ & & \\
\hline \multirow[t]{2}{*}{ Export South } & $0.004^{* * *}$ & $-0.006 * * *$ & & \\
\hline & $(0.001)$ & $(0.002)$ & & \\
\hline F stat & 3834.16 & 3834.16 & & \\
\hline $\mathrm{N}$ & 8,566 & 8,566 & & \\
\hline R-sq & 0.159 & 0.408 & & \\
\hline Step 2, skill definition 1 & Mean & SD & Mean & SD \\
\hline \multirow{2}{*}{ First year lag of predicted (w90/w50) } & $0.326^{* * *}$ & $0.102^{* * *}$ & & \\
\hline & $(0.114)$ & $(0.026)$ & & \\
\hline \multirow[t]{2}{*}{ First year lag of predicted (w50/w10) } & & & $-0.219^{* *}$ & $-0.068^{* *}$ \\
\hline & & & $(0.092)$ & $(0.031)$ \\
\hline $\mathrm{N}$ & 8,566 & 8,566 & 8,566 & 8,566 \\
\hline \multirow[t]{2}{*}{ R-sq } & 0.893 & 0.567 & 0.893 & 0.567 \\
\hline & \multicolumn{4}{|c|}{ Portugal } \\
\hline Step 1 & w90/w50 & w50/w10 & & \\
\hline \multirow[t]{2}{*}{ Export North } & 0.029 & 0.042 & & \\
\hline & $(0.023)$ & $(0.028)$ & & \\
\hline \multirow[t]{2}{*}{ Export South } & -0.005 & -0.036 & & \\
\hline & $(0.028)$ & $(0.035)$ & & \\
\hline F stat & 122.88 & 122.88 & & \\
\hline $\mathrm{N}$ & 1,102 & 1,102 & & \\
\hline R-sq & 0.549 & 0.328 & & \\
\hline Step 2, skill definition 1 & Mean & SD & Mean & SD \\
\hline First year lag of predicted (w90/w50) & omitted & omitted & & \\
\hline \multicolumn{3}{|l|}{ First year lag of predicted (w50/w10) } & omitted & omitted \\
\hline $\mathrm{N}$ & 1,042 & 1,042 & 1,042 & 1,042 \\
\hline R-sq & 0.966 & 0.756 & 0.966 & 0.756 \\
\hline
\end{tabular}

Notes: Skill definition 1 is based on the whole systematic component of the two-way fixed effects wage regression. In step 1, standard errors are clustered at the industry level. In step 2, standard errors are clustered at the industry level and sequentially bootstrapped with step 1, 200 replications. All estimations are also weighted for the number of firms in each industry. Both steps also include the following control variables: sales per employee, capital intensity (only for Denmark), workforce composition characteristics, year and 2 digit industry dummies. Significance levels: ${ }^{*} * * 1 \%,{ }^{*} 55 \%$, $* 10 \%$. 
Table 7: Effects of Trade Activity on the Skill Premium and Distribution, the role of imports.

\begin{tabular}{|c|c|c|c|c|}
\hline & \multicolumn{4}{|c|}{ Denmark } \\
\hline Step 1 & w90/w50 & $\mathrm{w} 50 / \mathrm{w} 10$ & & \\
\hline Import & $\begin{array}{c}0.004^{* * *} \\
(0.000)\end{array}$ & $\begin{array}{c}-0.008^{* * * *} \\
(0.001)\end{array}$ & & \\
\hline F stat & 31199.44 & 31199.44 & & \\
\hline $\mathrm{N}$ & 8,566 & 8,566 & & \\
\hline R-sq & 0.154 & 0.400 & & \\
\hline Step 2, skill definition 1 & Mean & SD & Mean & SD \\
\hline First year lag of predicted (w90/w50) & $\begin{array}{c}0.357^{* * *} \\
(0.122)\end{array}$ & $\begin{array}{c}0.190^{* * *} \\
(0.059)\end{array}$ & & \\
\hline First year lag of predicted (w50/w10) & & & $\begin{array}{c}-0.177^{* *} \\
(0.08)\end{array}$ & $\begin{array}{c}-0.094^{* *} \\
(0.046)\end{array}$ \\
\hline $\mathrm{N}$ & 8,566 & 8,566 & 8,566 & 8,566 \\
\hline \multirow[t]{2}{*}{$\mathrm{R}$-sq } & 0.893 & 0.567 & 0.893 & 0.567 \\
\hline & \multicolumn{4}{|c|}{ Portugal } \\
\hline Step 1 & w90/w50 & w50/w10 & & \\
\hline Import & $\begin{array}{c}0.042^{* * *} \\
(0.005)\end{array}$ & $\begin{array}{c}0.024^{* * *} \\
(0.005)\end{array}$ & & \\
\hline F stat & 281.17 & 281.17 & & \\
\hline $\mathrm{N}$ & 1,126 & 1,126 & & \\
\hline R-sq & 0.604 & 0.610 & & \\
\hline Step 2, skill definition 1 & Mean & SD & Mean & SD \\
\hline First year lag of predicted (w90/w50) & $\begin{array}{l}-0.016 \\
(0.023)\end{array}$ & $\begin{array}{c}0.036 \\
(0.029)\end{array}$ & & \\
\hline First year lag of predicted (w50/w10) & & & $\begin{array}{l}-0.027 \\
(0.047)\end{array}$ & $\begin{array}{c}0.061 \\
(0.045)\end{array}$ \\
\hline $\mathrm{N}$ & 1,064 & 1,064 & 1,064 & 1,064 \\
\hline R-sq & 0.920 & 0.667 & 0.920 & 0.667 \\
\hline
\end{tabular}

Notes: Skill definition 1 is based on the whole systematic component of the two-way fixed effects wage regression. In step 1, standard errors are clustered at the industry level. In step 2, standard errors are clustered at the industry level and sequentially bootstrapped with step 1, 200 replications. All estimations are also weighted for the number of firms in each industry. Both steps also include the following control variables: sales per employee, capital intensity (only for Denmark), workforce composition characteristics, year and 2 digit industry dummies. Significance levels: ***1\%, **5\%, $* 10 \%$. 
Table 8: Effects of Trade Activity on the Skill Premium and Distribution, high-tech sectors.

\begin{tabular}{|c|c|c|c|c|}
\hline & \multicolumn{4}{|c|}{ Denmark } \\
\hline Step 1 & w90/w50 & $\mathrm{w} 50 / \mathrm{w} 10$ & & \\
\hline \multirow[t]{2}{*}{ Import } & $0.004^{* *}$ & $-0.026^{* * *}$ & & \\
\hline & $(0.002)$ & $(0.007)$ & & \\
\hline F stat & 1285.377 & 1285.377 & & \\
\hline $\mathrm{N}$ & 1,313 & 1,313 & & \\
\hline R-sq & 0.255 & 0.472 & & \\
\hline Step 2, skill definition 1 & Mean & SD & Mean & SD \\
\hline \multirow[t]{2}{*}{ First year lag of predicted (w90/w50) } & $1.443^{* *}$ & $0.527^{* *}$ & & \\
\hline & $(0.578)$ & $(0.249)$ & & \\
\hline \multirow[t]{2}{*}{ First year lag of predicted (w50/w10) } & & & $-0.227^{* *}$ & $-0.083^{* *}$ \\
\hline & & & $(0.098)$ & $(0.039)$ \\
\hline $\mathrm{N}$ & 1,313 & 1,313 & 1,313 & 1,313 \\
\hline \multirow[t]{2}{*}{ R-sq } & 0.893 & 0.567 & 0.893 & 0.567 \\
\hline & \multicolumn{4}{|c|}{ Portugal } \\
\hline Step 1 & w90/w50 & w50/w10 & & \\
\hline \multirow[t]{2}{*}{ Export } & 0.012 & $0.051^{* * *}$ & & \\
\hline & $(0.007)$ & $(0.007)$ & & \\
\hline F stat & 83.945 & 83.945 & & \\
\hline $\mathrm{N}$ & 186 & 186 & & \\
\hline R-sq & 0.793 & 0.831 & & \\
\hline Step 2, skill definition 1 & Mean & SD & Mean & SD \\
\hline First year lag of predicted (w90/w50) & omitted & omitted & & \\
\hline \multirow[t]{2}{*}{ First year lag of predicted (w50/w10) } & & & $-0.044^{*}$ & -0.002 \\
\hline & & & $(0.024)$ & $(0.009)$ \\
\hline $\mathrm{N}$ & 176 & 176 & 176 & 176 \\
\hline R-sq & 0.957 & 0.746 & 0.975 & 0.746 \\
\hline
\end{tabular}

Notes: Skill definition 1 is based on the whole systematic component of the two-way fixed effects wage regression. In step 1, standard errors are clustered at the industry level. In step 2, standard errors are clustered at the industry level and sequentially bootstrapped with step 1, 200 replications. All estimations are also weighted for the number of firms in each industry. Both steps also include the following control variables: sales per employee, capital intensity (only for Denmark), workforce composition characteristics, year and 2 digit industry dummies. Significance levels: ***1\%, **5\%, $* 10 \%$. 
Table 9: Effects of Trade Activity on the Skill Premium and Distribution, low-tech sectors.

\begin{tabular}{|c|c|c|c|c|}
\hline & \multicolumn{4}{|c|}{ Denmark } \\
\hline Step 1 & w90/w50 & w50/w10 & & \\
\hline Import & $\begin{array}{c}0.009^{* * *} \\
(0.001)\end{array}$ & $\begin{array}{c}-0.033^{* * *} \\
(0.004)\end{array}$ & & \\
\hline F stat & 2820.041 & 2820.041 & & \\
\hline $\mathrm{N}$ & 6,157 & 6,157 & & \\
\hline R-sq & 0.173 & 0.377 & & \\
\hline Step 2, skill definition 1 & Mean & SD & Mean & SD \\
\hline First year lag of predicted (w90/w50) & $\begin{array}{c}0.344^{* *} \\
(0.156)\end{array}$ & $\begin{array}{l}0.081 \\
(0.06)\end{array}$ & & \\
\hline First year lag of predicted (w50/w10) & & & $\begin{array}{c}-0.091^{* * *} \\
(0.031)\end{array}$ & $\begin{array}{c}-0.021^{* *} \\
(0.009)\end{array}$ \\
\hline $\mathrm{N}$ & 6,157 & 6,157 & 6,157 & 6,157 \\
\hline \multirow[t]{2}{*}{ R-sq } & 0.893 & 0.567 & 0.893 & 0.567 \\
\hline & \multicolumn{4}{|c|}{ Portugal } \\
\hline Step 1 & w90/w50 & w50/w10 & & \\
\hline Export & $\begin{array}{c}0.049^{* * *} \\
(0.006)\end{array}$ & $\begin{array}{c}0.002 \\
(0.005)\end{array}$ & & \\
\hline F stat & 135.99 & 135.99 & & \\
\hline $\mathrm{N}$ & 940 & 940 & & \\
\hline R-sq & 0.433 & 0.638 & & \\
\hline Step 2, skill definition 1 & Mean & SD & Mean & SD \\
\hline First year lag of predicted (w90/w50) & $\begin{array}{c}0.0001 \\
(0.008)\end{array}$ & $\begin{array}{c}0.017^{*} \\
(0.009)\end{array}$ & & \\
\hline First year lag of predicted (w50/w10) & & & omitted & omitted \\
\hline $\mathrm{N}$ & 888 & 888 & 888 & 888 \\
\hline R-sq & 0.970 & 0.791 & 0.970 & 0.783 \\
\hline
\end{tabular}

Notes: Skill definition 1 is based on the whole systematic component of the two-way fixed effects wage regression. In step 1 , standard errors are clustered at the industry level. In step 2, standard errors are clustered at the industry level and sequentially bootstrapped with step 1, 200 replications. All estimations are also weighted for the number of firms in each industry. Both steps also include the following control variables: sales per employee, capital intensity (only for Denmark), workforce composition characteristics, year and 2 digit industry dummies. Significance levels: ***1\%, **5\%, $* 10 \%$. 
Table 10: Robustness check on the instrumental variable, the role of correlated business cycles across countries.

\begin{tabular}{|c|c|c|c|c|}
\hline & \multicolumn{4}{|c|}{ Denmark } \\
\hline Step 1 & w90/w50 & w50/w10 & & \\
\hline Export & $\begin{array}{c}0.005^{* * *} \\
(0.000)\end{array}$ & $\begin{array}{c}-0.007^{* * *} \\
(0.001)\end{array}$ & & \\
\hline F stat & 34768.76 & 34768.76 & & \\
\hline $\mathrm{N}$ & 8,566 & 8,566 & & \\
\hline R-sq & 0.1487427 & 0.3990634 & & \\
\hline Step 2, skill definition 1 & Mean & SD & Mean & SD \\
\hline First year lag of predicted (w90/w50) & $\begin{array}{c}1.646^{* * *} \\
(0.619)\end{array}$ & $\begin{array}{c}0.360^{* * *} \\
(0.133)\end{array}$ & & \\
\hline First year lag of predicted (w50/w10) & & & $\begin{array}{c}-1.015^{* * *} \\
(0.202)\end{array}$ & $\begin{array}{c}-0.118^{* * *} \\
(0.043)\end{array}$ \\
\hline $\mathrm{N}$ & 8,566 & 8,566 & 8,566 & 8,566 \\
\hline \multirow[t]{2}{*}{ R-sq } & 0.893 & 0.567 & 0.893 & 0.567 \\
\hline & \multicolumn{4}{|c|}{ Portugal } \\
\hline Step 1 & w90/w50 & w50/w10 & & \\
\hline Export & $\begin{array}{c}0.036^{* * *} \\
(0.004)\end{array}$ & $\begin{array}{c}0.019^{* * *} \\
(0.004)\end{array}$ & & \\
\hline F stat & 209.547 & 209.547 & & \\
\hline $\mathrm{N}$ & 1,137 & 1,137 & & \\
\hline R-sq & 0.555 & 0.598 & & \\
\hline Step 2, skill definition 1 & Mean & SD & Mean & SD \\
\hline First year lag of predicted (w90/w50) & $\begin{array}{l}-0.015 \\
(0.011)\end{array}$ & $\begin{array}{c}0.009 \\
(0.007)\end{array}$ & & \\
\hline First year lag of predicted (w50/w10) & & & $\begin{array}{c}-0.028 \\
(0.021)\end{array}$ & $\begin{array}{c}0.017 \\
(0.015)\end{array}$ \\
\hline $\mathrm{N}$ & 1,064 & 1,064 & 1,064 & 1,064 \\
\hline R-sq & 0.966 & 0.754 & 0.966 & 0.754 \\
\hline
\end{tabular}

Notes: Skill definition 1 is based on the whole systematic component of the two-way fixed effects wage regression. In step 1, standard errors are clustered at the industry level. In step 2, standard errors are clustered at the industry level and sequentially bootstrapped with step 1, 200 replications. All estimations are also weighted for the number of firms in each industry. Both steps also include the following control variables: sales per employee, capital intensity (only for Denmark), workforce composition characteristics, year and 2 digit industry dummies. Significance levels: ***1\%, **5\%, $* 10 \%$. 
Table 11: Robustness check on the instrumental variable: the role of correlated technology or demand shocks (Colantone and Crino', 2014)

\begin{tabular}{|c|c|c|c|c|}
\hline & \multicolumn{4}{|c|}{ Denmark } \\
\hline Step 1 & w90/w50 & w50/w10 & & \\
\hline Export & $\begin{array}{c}0.002^{* * *} \\
(0.000)\end{array}$ & $\begin{array}{c}-0.005^{* * *} \\
(0.001)\end{array}$ & & \\
\hline F stat & 29370.04 & 29370.04 & & \\
\hline $\mathrm{N}$ & 7,415 & 7,415 & & \\
\hline R-sq & 0.159 & 0.412 & & \\
\hline Step 2, skill definition 1 & Mean & SD & Mean & SD \\
\hline First year lag of predicted (w90/w50) & $\begin{array}{c}0.688^{* *} \\
(0.304)\end{array}$ & $\begin{array}{c}0.158^{* *} \\
(0.065)\end{array}$ & & \\
\hline First year lag of predicted (w50/w10) & & & $\begin{array}{c}-0.291^{* *} \\
(0.202)\end{array}$ & $\begin{array}{c}-0.067 \\
(0.042)\end{array}$ \\
\hline $\mathrm{N}$ & 7,415 & 7,415 & 7,415 & 7,415 \\
\hline \multirow[t]{2}{*}{ R-sq } & 0.893 & 0.567 & 0.893 & 0.567 \\
\hline & \multicolumn{4}{|c|}{ Portugal } \\
\hline Step 1 & w90/w50 & w50/w10 & & \\
\hline Export & $\begin{array}{c}0.037^{* * *} \\
(0.004)\end{array}$ & $\begin{array}{c}0.004 \\
(0.004)\end{array}$ & & \\
\hline F stat & 275.67 & 275.67 & & \\
\hline $\mathrm{N}$ & 979 & 979 & & \\
\hline R-sq & 0.584 & 0.675 & & \\
\hline Step 2, skill definition 1 & Mean & SD & Mean & SD \\
\hline First year lag of predicted (w90/w50) & $\begin{array}{l}-0.013 \\
(0.012)\end{array}$ & $\begin{array}{l}-0.006 \\
(0.005)\end{array}$ & & \\
\hline First year lag of predicted (w50/w10) & & & omitted & omitted \\
\hline $\mathrm{N}$ & 925 & 925 & 925 & 925 \\
\hline R-sq & 0.966 & 0.779 & 0.966 & 0.777 \\
\hline
\end{tabular}

Notes: Skill definition 1 is based on the whole systematic component of the two-way fixed effects wage regression. In step 1 , standard errors are clustered at the industry level. In step 2, standard errors are clustered at the industry level and sequentially bootstrapped with step 1, 200 replications. All estimations are also weighted for the number of firms in each industry. Both steps also include the following control variables: sales per employee, capital intensity (only for Denmark), workforce composition characteristics, year and 2 digit industry dummies. Significance levels: ***1\%,**5\%, $* 10 \%$. 
Table 12: Robustness check on instrumental variable: the role of correlated technology or demand shocks (Autor et al., 2013)

\begin{tabular}{|c|c|c|c|c|}
\hline & \multicolumn{4}{|c|}{ Denmark } \\
\hline Step 1 & w90/w50 & w50/w10 & & \\
\hline Export & $\begin{array}{c}0.002^{* * *} \\
(0.000)\end{array}$ & $\begin{array}{c}-0.005^{* * *} \\
(0.001)\end{array}$ & & \\
\hline F stat & 27887.92 & 27887.92 & & \\
\hline $\mathrm{N}$ & 6,866 & 6,866 & & \\
\hline R-sq & 0.164 & 0.414 & & \\
\hline Step 2, skill definition 1 & Mean & SD & Mean & SD \\
\hline First year lag of predicted (w90/w50) & $\begin{array}{c}0.540^{* *} \\
(0.249)\end{array}$ & $\begin{array}{l}0.119^{*} \\
(0.069)\end{array}$ & & \\
\hline First year lag of predicted (w50/w10) & & & $\begin{array}{c}-0.251^{* *} \\
(0.106)\end{array}$ & $\begin{array}{c}-0.055^{* *} \\
(0.023)\end{array}$ \\
\hline $\mathrm{N}$ & 6,866 & 6,866 & 6,866 & 6,866 \\
\hline \multirow[t]{2}{*}{ R-sq } & 0.893 & 0.597 & 0.893 & 0.597 \\
\hline & \multicolumn{4}{|c|}{ Portugal } \\
\hline Step 1 & w90/w50 & w50/w10 & & \\
\hline Export & $\begin{array}{c}0.026^{* * *} \\
(0.004)\end{array}$ & $\begin{array}{c}0.025^{* * *} \\
(0.005)\end{array}$ & & \\
\hline F stat & 166.11 & 166.11 & & \\
\hline $\mathrm{N}$ & 921 & 921 & & \\
\hline R-sq & 0.647 & 0.512 & & \\
\hline Step 2, skill definition 1 & Mean & SD & Mean & SD \\
\hline First year lag of predicted (w90/w50) & $\begin{array}{c}-0.036^{*} \\
(0.020)\end{array}$ & $\begin{array}{c}0.008 \\
(0.010)\end{array}$ & & \\
\hline First year lag of predicted (w50/w10) & & & $\begin{array}{l}-0.035 \\
(0.029)\end{array}$ & $\begin{array}{c}0.009 \\
(0.013)\end{array}$ \\
\hline $\mathrm{N}$ & 870 & 870 & 870 & 870 \\
\hline R-sq & 0.966 & 0.766 & 0.966 & 0.766 \\
\hline
\end{tabular}

Notes: Skill definition 1 is based on the whole systematic component of the two-way fixed effects wage regression. In step 1 , standard errors are clustered at the industry level. In step 2, standard errors are clustered at the industry level and sequentially bootstrapped with step 1, 200 replications. All estimations are also weighted for the number of firms in each industry. Both steps also include the following control variables: sales per employee, capital intensity (only for Denmark), workforce composition characteristics, year and 2 digit industry dummies. Significance levels: ***1\%, **5\%, $* 10 \%$. 
Table 13: Effects of Trade Activity on the Skill Premium and Distribution, Danish industry classification at 3 -digit.

\begin{tabular}{|c|c|c|c|c|}
\hline & \multicolumn{4}{|c|}{ Denmark } \\
\hline Step 1 & w90/w50 & w50/w10 & & \\
\hline Export & $\begin{array}{c}0.002^{* * *} \\
(0.001)\end{array}$ & $\begin{array}{c}-0.011^{* * *} \\
(0.002)\end{array}$ & & \\
\hline F stat & 8061.727 & 8061.727 & & \\
\hline $\mathrm{N}$ & 1,200 & 1,200 & & \\
\hline R-sq & 0.253 & 0.452 & & \\
\hline Step 2, skill definition 1 & Mean & SD & Mean & SD \\
\hline Lagged of predicted (w90/w50) & $\begin{array}{c}1.896^{* * *} \\
(0.873)\end{array}$ & $\begin{array}{c}0.705 * * \\
(0.353)\end{array}$ & & \\
\hline Lagged of predicted (w50/w10) & & & $\begin{array}{c}-1.106^{* * *} \\
(0.183)\end{array}$ & $\begin{array}{c}-0.132^{* *} \\
(0.066)\end{array}$ \\
\hline $\mathrm{N}$ & 1,200 & 1,200 & 1,200 & 1,200 \\
\hline R-sq & 0.893 & 0.567 & 0.893 & 0.567 \\
\hline
\end{tabular}

Notes: Skill definition 1 is based on the whole systematic component of the two-way fixed effects wage regression. Both steps also include the following control variables: sales per employee, capital intensity, workforce composition characteristics, year and 2 digit industry dummies. In step 1, standard errors are clustered at the industry level. In step 2, standard errors are clustered at the industry level and sequentially bootstrapped with step 1, 200 replications. All estimations are also weighted for the number of firms in each industry. Significance levels: $* * * 1 \%, * * 5 \%,{ }^{*} 10 \%$. 


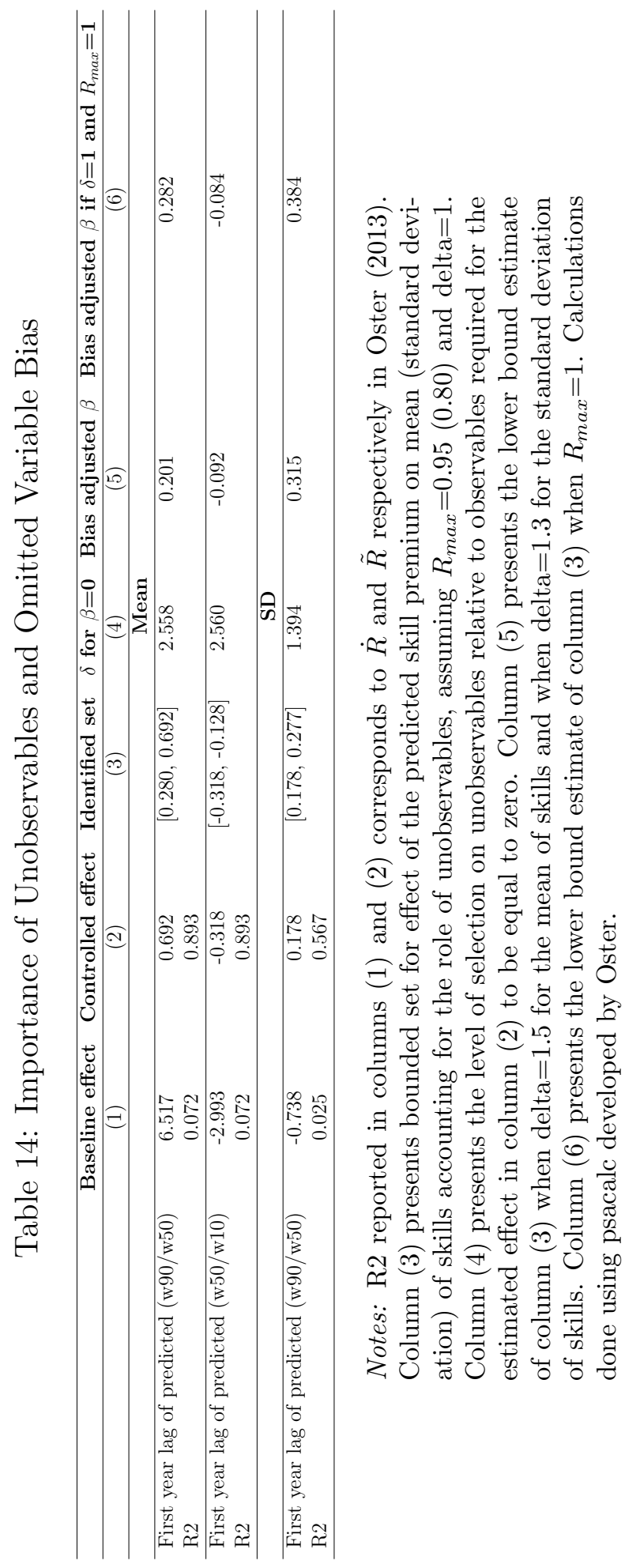


Table 15: Effects of Trade Activity on the Skill Premium and Distribution, specification with two and three years lag.

\begin{tabular}{|c|c|c|c|c|}
\hline & \multicolumn{4}{|c|}{ Denmark } \\
\hline Step 2, skill definition 1 & Mean & SD & Mean & SD \\
\hline Predicted (w90/w50), two years lag & $\begin{array}{c}0.631^{* *} \\
(0.281)\end{array}$ & $\begin{array}{l}0.176^{*} \\
(0.101)\end{array}$ & & \\
\hline Predicted (w50/w10), two years lag & & & $\begin{array}{c}-0.290^{* * *} \\
(0.141)\end{array}$ & $\begin{array}{r}-0.081^{*} \\
(0.048)\end{array}$ \\
\hline $\mathrm{N}$ & 8,046 & 8,046 & 8,046 & 8,046 \\
\hline R-sq & 0.793 & 0.547 & 0.793 & 0.544 \\
\hline Step 2, skill definition 1 & Mean & SD & Mean & SD \\
\hline Predicted (w90/w50), three years lag & $\begin{array}{c}0.453 \\
(0.313)\end{array}$ & $\begin{array}{c}0.207^{* * *} \\
(0.064)\end{array}$ & & \\
\hline Predicted (w50/w10), three years lag & & & $\begin{array}{c}-0.208^{*} \\
(0.113)\end{array}$ & $\begin{array}{c}-0.095 \\
(0.071)\end{array}$ \\
\hline $\mathrm{N}$ & 8,046 & 8,046 & 8,046 & 8,046 \\
\hline \multirow[t]{2}{*}{ R-sq } & 0.761 & 0.512 & 0.772 & 0.532 \\
\hline & \multicolumn{4}{|c|}{ Portugal } \\
\hline Step 2, skill definition 1 & Mean & $\mathrm{SD}$ & Mean & $\mathrm{SD}$ \\
\hline Predicted (w90/w50), two years lag & $\begin{array}{l}-0.015 \\
(0.012)\end{array}$ & $\begin{array}{c}0.006 \\
(0.008)\end{array}$ & & \\
\hline Predicted (w50/w10), two years lag & & & $\begin{array}{c}-0.027 \\
(0.022)\end{array}$ & $\begin{array}{c}0.012 \\
(0.017) \\
\end{array}$ \\
\hline $\mathrm{N}$ & 1,003 & 1,003 & 1,003 & 1,003 \\
\hline R-sq & 0.964 & 0.760 & 0.964 & 0.760 \\
\hline Step 2, skill definition 1 & Mean & SD & Mean & SD \\
\hline Predicted (w90/w50), three years lag & $\begin{array}{l}-0.020 \\
(0.014)\end{array}$ & $\begin{array}{c}0.004 \\
(0.008)\end{array}$ & & \\
\hline Predicted (w50/w10), three years lag & & & $\begin{array}{c}-0.036 \\
(0.025)\end{array}$ & $\begin{array}{c}0.007 \\
(0.015)\end{array}$ \\
\hline $\mathrm{N}$ & 942 & 942 & 942 & 942 \\
\hline R-sq & 0.962 & 0.765 & 0.962 & 0.765 \\
\hline
\end{tabular}

Notes: Skill definition 1 is based on the whole systematic component of the two-way fixed effects wage regression. Both steps also include the following control variables: sales per employee, capital intensity (only for Denmark), workforce composition characteristics, year and 2 digit industry dummies. In step 1, standard errors are clustered at the industry level. In step 2, standard errors are clustered at the industry level and sequentially bootstrapped with step 1, 200 replications. All estimations are also weighted for the number of firms in each industry. Significance levels: $* * * 1 \%$, $* * 5 \%, * 10 \%$. 